\title{
Gravitational Lensing of Charged Ayon-Beato-Garcia Black Holes and Nonlinear Effects of Maxwell Fields
}

\author{
H. Ghaffarnejad $(\mathbb{D}$, M. Amirmojahedi, and H. Niad \\ Faculty of Physics, Semnan University, Semnan 35131-19111, Iran \\ Correspondence should be addressed to H. Ghaffarnejad; hghafarnejad@semnan.ac.ir
}

Received 6 July 2017; Accepted 22 January 2018; Published 12 March 2018

Academic Editor: Andrea Coccaro

Copyright (C) 2018 H. Ghaffarnejad et al. This is an open access article distributed under the Creative Commons Attribution License, which permits unrestricted use, distribution, and reproduction in any medium, provided the original work is properly cited. The publication of this article was funded by $\mathrm{SCOAP}^{3}$.

\begin{abstract}
Nonsingular Ayon-Beato-Garcia (ABG) spherically symmetric static black hole $(\mathrm{BH})$ with charge to mass ratio $q=g / 2 \mathrm{~m}$ is metric solution of Born Infeld nonlinear Maxwell-Einstein theory. Central region of the BH behaves as (anti-)de Sitter for $(|q|>1)|q|<$ 1. In the case where $|q|=1$, the BH central region behaves as Minkowski flat metric. Nonlinear Electromagnetic (NEM) fields counterpart causes deviation of light geodesics and so light rays will be forced to move on from effective metric. In this paper we study weak and strong gravitational lensing of light rays by seeking effects of NEM fields counterpart on image locations and corresponding magnification. We set our calculations to experimentally observed Sgr A* $\mathrm{BH}$. In short we obtained the following: for large distances, the NEM counterpart is negligible and it reduces to linear Maxwell fields. The NEM field enlarges radius of the $\mathrm{BH}$ photon sphere linearly by raising $|q|>1$ but decreases by raising $|q| \leq 1$. Sign of deflection angle of bending light rays is changed in presence of NEM effects with respect to ones obtained in absence of NEM fields. Absolute value of deflection angle rises by increasing $|q| \rightarrow 1$. Image locations in weak deflection limit (WDL) decrease (increases) by raising $0<|q|<1$ in presence (absence) of NEM fields. By raising the closest distance of the bending light rays image locations in WDL change from left (right) to right (left) in absence (presence) of NEM fields. In WDL, radius of Einstein rings and corresponding magnification centroid become larger (smaller) in presence (absence) of NEM fields. Angular separation called $s$ between the innermost and outermost relativistic images increases (decreases) by increasing $0<|q|<1$ in absence (presence) of NEM fields. Corresponding magnification $r$ decreases (increases) by raising $0<|q|<1$ in absence (presence) of NEM fields.
\end{abstract}

\section{Introduction}

Since the advent of Einstein's general relativity theory, black holes and the singularity problem of curved space-time become challenging subjects in modern (quantum) physics. Singularity is the intrinsic character of the most exact solutions of Einstein's equations where Ricci and Kretschmann scalars reach to infinite value at singular point of the spacetime [1]. Penrose cosmic censorship conjecture states that the causal singularities must be covered by the event horizon and so causes disconnecting of interior and exterior regions of the space-time $[2,3]$. On the other side back-reaction corrections of quantum matter field cause shrinking of the quantum black hole horizon (see [4] and references therein) for which the Penrose cosmic censorship may become invalid for quantum regimes. At the moment one should infer that final state of evaporating quantum black holes is still open problem. This encourages one to seek nonsingular type of black hole solutions of the Einstein's equation. However nonsingular metric solutions are also obtained from the Einstein field equation [5-26]. In the latter situations the Einstein field equation is coupled with suitable NEM fields for which the Ricci and the Kretschmann scalars become regular in whole space-time. A good classification of spherically symmetric static regular black holes is collected in [10]. Inspiring a physical central core idea, Bardeen (BAR) suggested the first spherically symmetric static regular black hole in 1968 containing a horizon without singularity [11]. After his work, other regular black holes were designed based on this model which we call here, for instance, ABG [12-15], Hayward (HAY) [16], and Neves-Saa (NS) [17, 18]. Nonsingular property of all of these solutions is controlled via dimensionless charge parameter $q$. HAY type of regular black hole is obtained by modifying the mass parameter of the BAR 
black hole. NS type of regular black hole is similar to a HAY type but its asymptotic behavior approaches to a vacuum de Sitter in presence of cosmological constant parameter. Regular black holes are studied also on brane words (see [18] and reference therein). The solutions of rotating regular black holes have been introduced in several articles [19-27]. A very important gravitational source is the Kerr-Newman(anti) de Sitter (KNdS/KNAdS) black hole which is studied first by Stuchlík in [28] (see also [29, 30]). Kraniotis studied gravitational lensing of KNdS and KNAdS black hole in [31], where closed form analytic solutions of the null geodesics and the gravitational lens equations have been obtained versus the Appell-Lauricella generalized hypergeometric functions and the elliptic functions of Weierstrass. In these exact solutions all the fundamental parameters of the theory, namely, black hole mass, electric charge, rotation angular momentum, and the cosmological constant, enter on an equal footing while the electric charge effect on relativistic observable was also investigated. Rotating nonsingular black holes can be treat as natural particle accelerators [27]. Ultrahigh energy particle collisions are studied on the regular black holes [32] and backgrounds containing naked singularity [33]. Motion of test particles is studied in regular black hole space-time in [34]. Circular geodesics are obtained for BAR and ABG regular black holes in [35]. The optical effects related to Keplerian discs orbiting Kehagias-Sfetsos (KS) naked singularities were investigated in [36]. Authors of the latter work also mentioned the close similarity between circular geodesics in KS and properties of the circular geodesics of the RN naked singular space-time. Schee and Stuchlík studied also profiled spectral lines generated by Keplerian discs orbiting in the BAR and the ABG space-time in [37]. Correspondence between the black holes and the FRW geometries is studied for nonrelativistic gravity models in [38]. Reissner Nordström (RN) black hole gravitational lensing is studied in [39]. Gravitational lensing from regular black holes is studied in WDL (SDL) of light rays in [4047]. Strong deflection limits of light rays can distinguish gravitational lensing between naked singularity and regular black holes background [47]. There is significant difference between optical phenomena characters of the singular spacetimes such as Schwarzschild (SCH), RN, and nonsingular space-times as HAY, BAR, and ABG [44]. It is related to the fact that central regions of the regular space-times reach to de Sitter (anti-de Sitter) for $|q|<1(|q|>1)$ (see (7) and (9)). A detailed analysis of the null geodesic motion of the original regular space-time and comparison to the RN space-time is presented by Genzel et al. [48]. Furthermore we should point that the nonsingular charged black holes obtained from NEM models in curved space-time cause the photons to not move along null geodesics. As an applicable approach we should obtain corresponding effective metric for geodesics of moving photons [49-52] and then proceed to study their gravitational lensing. The black hole electric charge has also important effects on final state of the Hawking radiation and switching off effects of a quantum evaporating black hole (see, e.g., [53]). In this work we study gravitational lensing of light rays moving on the $\mathrm{ABG}$ nonsingular black hole in presence of NEM fields counterparts. The paper is organized as follows.
Briefly, we introduce regular ABG black hole metric in Section 2 and its asymptotically behavior for different values of $q$. In Section 3 we calculate effective metric of the ABG black hole for the moving photons by regarding the results of the original work [13]. We solve numerically the photon sphere equation of effective metric and obtain photon sphere radius against different charge values for $q$. In Section 4 we evaluate general formalism of deflection angle of bending light rays in weak and strong deflection limits. In weak deflection limits we apply the Ohanian lens equation [54] to determine nonrelativistic image locations versus source positions for observed Sgr A* black hole [48, 55-57]. Weak deflection angle of bending light rays and their magnifications are evaluated numerically point by point and they are plotted against source locations and also $q$. In the strong deflection limits we use Bozza's formalism [43, $44]$ to obtain logarithmic form of the deflection angle. We calculate relative distance between innermost and outermost relativistic images and corresponding magnification and then plot their diagrams. Section 5 denotes the concluding remark.

\section{ABG Space-Time}

The ABG spherically symmetric black hole metric defined by Schwarzschild coordinates is given by [13]

$$
d s^{2}=-H(r) d t^{2}+\frac{d r^{2}}{H(r)}+r^{2}\left(d \theta^{2}+\sin ^{2} \theta d \varphi^{2}\right)
$$

with

$$
H(r)=1-\frac{2 m r^{2}}{\left(r^{2}+g^{2}\right)^{3 / 2}}+\frac{g^{2} r^{2}}{\left(r^{2}+g^{2}\right)^{2}}
$$

and associated electric field

$$
F_{t r}(r)=E(r)=g r^{4}\left(\frac{r^{2}-5 g^{2}}{\left(r^{2}+g^{2}\right)^{4}}+\frac{15}{2} \frac{m}{\left(r^{2}+g^{2}\right)^{7 / 2}}\right) .
$$

$m$ and $g$ are total mass and electric charge parameters of the $\mathrm{ABG} \mathrm{BH}$, respectively. The line element (1) is nonsingular static solution of NEM-Einstein metric equation

$$
G_{\mu \nu}=8 \pi T_{\mu \nu}=8 \pi\left\{\mathscr{L}_{F} F_{\mu \eta} F_{\nu}^{\eta}-\mathscr{L}_{\mu \nu}\right\}, \quad \mathscr{L}_{F}=\frac{\partial \mathscr{L}}{\partial F}
$$

which satisfies the action functional $I=\int \sqrt{g} d x^{4}(R / 16 \pi-$ $\mathscr{L}(F) / 4 \pi$ ) where $R$ is Ricci scalar and $\mathscr{L}$ is a functional of $F=(1 / 4) F_{\mu \nu} F^{\mu \nu}$. This metric solution has only the coordinate singularity called horizon singularity without causal singularity called event horizon singularity because the Ricci $R_{\mu}^{\mu}$ and the Kretschmann $R^{\mu \nu \alpha \lambda} R_{\mu \nu \alpha \lambda}$ scalars become regular at all points of the space-time $0 \leq r \leq+\infty$. Defining mass and charge functions as

$$
\begin{aligned}
& M(r)=m\left(1+\frac{g^{2}}{r^{2}}\right)^{-3 / 2}, \\
& e(r)=g\left(1+\frac{g^{2}}{r^{2}}\right)^{-1}
\end{aligned}
$$


one can show that the $A B G$ metric (1) reduces apparently to a variable mass-charge $\mathrm{RN}$ type of $\mathrm{BH}$ as follows.

$$
\begin{aligned}
d s^{2}= & -\left(1-\frac{2 M(r)}{r}+\frac{e^{2}(r)}{r^{2}}\right) d t^{2} \\
& +\frac{d r^{2}}{\left(1-2 M(r) / r+e^{2}(r) / r^{2}\right)} \\
& +r^{2}\left(d \theta^{2}+\sin ^{2} \theta d \varphi^{2}\right),
\end{aligned}
$$

where $M(\infty)=m$ and $e^{2}(\infty)=g$ are ADM mass and electric charge viewed from observer located at infinity. Its central region $0<r<|g|$ behaves as vacuum de Sitter asymptotically:

$$
\begin{aligned}
d s^{2} \approx & -\left(1-\frac{\Lambda}{3} r^{2}\right) d t^{2}+\frac{d r^{2}}{\left(1-(\Lambda / 3) r^{2}\right)} \\
& +r^{2}\left(d \theta^{2}+\sin ^{2} \theta d \varphi^{2}\right)
\end{aligned}
$$

for

$$
|q|=\frac{g}{2 m}<1
$$

and anti-de Sitter

$$
\begin{aligned}
d s^{2} \approx & -\left(1+\frac{\Lambda}{3} r^{2}\right) d t^{2}+\frac{d r^{2}}{\left(1+(\Lambda / 3) r^{2}\right)} \\
& +r^{2}\left(d \theta^{2}+\sin ^{2} \theta d \varphi^{2}\right)
\end{aligned}
$$

for

$$
|q|=\frac{g}{2 m}>1
$$

respectively, where we defined effective cosmological constant as

$$
\Lambda(m, g)=\frac{3(1-|q|)}{4 m^{2} q^{3}}
$$

In the particular case

$$
|q|=\frac{g}{2 m}=1
$$

the effective cosmological parameter vanishes, $\Lambda=0$, and so, near the center $r \rightarrow 0$, the ABG black hole metric reduces to a flat Minkowski background asymptotically. Setting $g=0$, (5) read $m=M, e=0$ for which the metric solution (1) leads to singular charge-less Schwarzschild BH. Nonlinear counterpart of the Maxwell stress tensor causes deviation of the photon geodesics where the photons do not move along the null geodesics. Usually one uses an effective metric to study gravitational lensing of the light rays moving on such a charged black holes metric $[47,49-51,58]$. In the following section we seek effective metric of the ABG black hole for photon trajectories.

\section{Effective Metric for Photon Trajectories}

Assuming $\mathscr{L}(F)=F$, (4) leads to the well known linear Einstein-Maxwell gravity where the photon propagates by the null equation

$$
g_{\mu \nu} k^{\mu} k^{\nu}=0
$$

where $k^{\mu}$ is corresponding four-momentum of the photon, but in general form where $\mathscr{L}(F) \neq F$ the electric field given by (3) is self-interacting and so directly is reflected on the photon propagation. In the latter case the photons do not move along null geodesics of background geometry and so (13) becomes invalid. Instead, the photons will be propagate along null geodesics of an effective geometry which depends on the NEM source for which we will have $[50,51,59]$ :

$$
g_{\mu \nu}^{\mathrm{eff}} k^{\mu} k^{\nu}=0
$$

where

$$
\begin{aligned}
& g_{\mu \nu}^{\mathrm{eff}}=16\left(\frac{\mathscr{L}_{F F} F_{\mu \eta} F_{v}^{\eta}-\left(\mathscr{L}_{F}+2 F \mathscr{L}_{F F}\right) g_{\mu \nu}}{F^{2} \mathscr{L}_{F F}^{2}-16\left(\mathscr{L}_{F}+F \mathscr{L}_{F F}\right)^{2}}\right), \\
& g_{\mathrm{eff}}^{\mu \nu}=\mathscr{L}_{F F} F_{\eta}^{\mu} F^{\nu \eta}+\mathscr{L}_{F} g^{\mu \nu} .
\end{aligned}
$$

In absence of nonlinear counterpart of EM fields we can infer

$$
\begin{gathered}
\mathscr{L}_{F F}=0, \\
\mathscr{L}_{F}=1, \\
\mathscr{L}=F
\end{gathered}
$$

for which $g_{\mu \nu}^{\text {eff }} \rightarrow g_{\mu \nu}$. Now we are in a position to obtain exact form for the effective metric of spherically symmetric static space-time (1). To do so we need to calculate all quantities defined by $\left\{\mathscr{L}, \mathscr{L}_{F}, \mathscr{L}_{F F}\right\}$ which satisfy the metric solution (1). We should first obtain corresponding Lagrangian density $\mathscr{L}(F)$. Authors of the original paper [13] used the following ansatz to solve (4) and to obtain (1).

$$
F \mathscr{L}_{F}^{2}=-\frac{1}{2(2 m)^{2}} \frac{q^{2}}{x^{4}},
$$

where

$$
\begin{aligned}
& F(x) \\
& \quad=-\frac{1}{(2 m)^{2}} \frac{q^{2} x^{8}}{2}\left[\frac{x^{2}-5 q^{2}}{\left(x^{2}+q^{2}\right)^{4}}+\frac{15}{4} \frac{1}{\left(x^{2}+q^{2}\right)^{7 / 2}}\right]^{2} .
\end{aligned}
$$

One can obtain the above equation by substituting the following dimensionless parameters into the EM Lagrangian density $F=(1 / 4) F_{\mu \nu} F^{\mu \nu}$.

$$
\begin{aligned}
& q=\frac{g}{2 m}, \\
& x=\frac{r}{2 m} .
\end{aligned}
$$


TABLE 1: Numerical major real roots of the photon sphere equations (26) and (28) for $0<|q|<1$.

\begin{tabular}{|c|c|c|c|}
\hline$|q|, x_{p s}, x_{p s}^{\mathrm{eff}}$ & $|q|, x_{p s}, x_{p s}^{\mathrm{eff}}$ & $|q|, x_{p s}, x_{p s}^{\text {eff }}$ & $|q|, x_{p s}, x_{p s}^{\text {eff }}$ \\
\hline $0.00,3.000,3.000$ & $0.25,2.892,2.874$ & $0.50,2.547,2.645$ & $0.75,-, 2.326$ \\
\hline $0.01,2.999,2.950$ & $0.26,2.887,2.871$ & $0.51,2.528,2.640$ & $0.76,-, 2.320$ \\
\hline $0.02,2.994,2.947$ & $0.27,2.882,2.865$ & $0.52,2.508,2.630$ & $0.77,-, 2.326$ \\
\hline $0.03,2.989,2.947$ & $0.28,2.872,2.855$ & $0.53,2.475,2.621$ & $0.78,-, 2.329$ \\
\hline $0.04,2.989,2.947$ & $0.29,2.858,2.852$ & $0.54,2.455,2.609$ & $0.79,-, 2.335$ \\
\hline $0.05,2.989,2.944$ & $0.30,2.848,2.843$ & $0.55,2.431,2.594$ & $0.80,-, 2.344$ \\
\hline $0.06,2.989,2.943$ & $0.31,2.838,2.831$ & $0.56,2.397,2.578$ & $0.81,-, 2.350$ \\
\hline $0.07,2.984,2.942$ & $0.32,2.828,2.828$ & $0.57,2.378,2.560$ & $0.82,-, 2.362$ \\
\hline $0.08,2.980,2.941$ & $0.33,2.824,2.819$ & $0.58,2.334,2.530$ & $0.83,-, 2.377$ \\
\hline $0.09,2.980,2.938$ & $0.34,2.809,2.813$ & $0.59,2.300,2.514$ & $0.84,-, 2.393$ \\
\hline $0.10,2.975,2.935$ & $0.35,2.795,2.804$ & $0.60,2.271,2.508$ & $0.85,-, 2.408$ \\
\hline $0.11,2.974,2.935$ & $0.36,2.785,2.795$ & $0.61,2.227,2.478$ & $0.86,-, 2.429$ \\
\hline $0.12,2.974,2.935$ & $0.37,2.770,2.785$ & $0.62,2.193,2.469$ & $0.87,-, 2.451$ \\
\hline $0.13,2.964,2.932$ & $0.38,2.756,2.776$ & $0.63,2.150,2.460$ & $0.88,-, 2.475$ \\
\hline $0.14,2.959,2.928$ & $0.39,2.736,2.767$ & $0.64,2.106,2.441$ & $0.89,-, 2.487$ \\
\hline $0.15,2.955,2.925$ & $0.40,2.722,2.758$ & $0.65,2.058,2.429$ & $0.90,-, 2.514$ \\
\hline $0.16,2.950,2.919$ & $0.41,2.707,2.749$ & $0.66,1.990,2.411$ & $0.91,-, 2.542$ \\
\hline $0.17,2.945,2.916$ & $0.42,2.698,2.740$ & $0.67,1.902,2.399$ & $0.92,-, 2.566$ \\
\hline $0.18,2.940,2.913$ & $0.43,2.678,2.731$ & $0.68,1.819,2.384$ & $0.93,-, 2.591$ \\
\hline $0.19,2.935,2.910$ & $0.44,2.659,2.718$ & $0.69,1.645,2.374$ & $0.94,-, 2.612$ \\
\hline $0.20,2.930,2.904$ & $0.45,2.654,2.706$ & $0.70,-, 2.362$ & $0.95,-, 2.636$ \\
\hline $0.21,2.921,2.989$ & $0.46,2.630,2.694$ & $0.71,-, 2.350$ & $0.96,-, 2.670$ \\
\hline $0.22,2.916,2.895$ & $0.47,2.601,2.685$ & $0.72,-, 2.344$ & $0.97,-, 2.703$ \\
\hline $0.23,2.906,2.889$ & $0.48,2.591,2.670$ & $0.73,-, 2.338$ & $0.98,-, 2.734$ \\
\hline $0.24,2.901,2.883$ & $0.49,2.557,2.658$ & $0.74,-, 2.335$ & $0.99,-, 2.764$ \\
\hline
\end{tabular}

Equation (19) behaves asymptotically as $F_{\infty}(x) \approx-(1 /$ $\left.2(2 m)^{2}\right)\left(q^{2} / x^{4}\right)$ for large distances $x \gg 1$. Comparing the latter result and (18) we can infer $\mathscr{L}_{F} \approx 1$ for large distances $x \gg 1$. One can integrate it to obtain linear Maxwell Lagrangian $\mathscr{L} \rightarrow F$. The latter result tells us NEM action functionals $\mathscr{L}(F)$ are negligible for regions of far from the black hole event horizon $x \gg x_{E H}$. Applying (18) and (19) we obtain parametric form of the Lagrangian density $\mathscr{L}(x)$ as follows.

$$
\begin{aligned}
\mathscr{L}(x)= & -\frac{q^{2}}{(2 m)^{2}} \int_{\infty}^{x} \frac{1}{x^{2}} d \\
& \cdot\left\{x^{4}\left[\frac{x^{2}-5 q^{2}}{\left(x^{2}+q^{2}\right)^{4}}+\frac{15}{4} \frac{1}{\left(x^{2}+q^{2}\right)^{7 / 2}}\right]\right\}
\end{aligned}
$$

which has exact solution as

$$
\begin{aligned}
& \mathscr{L}(x)=-\frac{q^{2}}{(2 m)^{2}}\left[\frac{x^{2}\left(x^{2}-5 q^{2}\right)}{\left(x^{2}+q^{2}\right)^{4}}+\frac{15}{4} \frac{x^{2}}{\left(x^{2}+q^{2}\right)^{7 / 2}}\right] \\
& +\frac{q^{2}}{(2 m)^{2}}\left[\frac{1}{2\left(x^{2}+q^{2}\right)^{2}}-\frac{2 q^{2}}{\left(x^{2}+q^{2}\right)^{3}}\right. \\
& \left.+\frac{3}{2} \frac{1}{\left(x^{2}+q^{2}\right)^{5 / 2}}\right] .
\end{aligned}
$$

One can infer

$$
\begin{aligned}
\mathscr{L}_{F}(x) & =\frac{\mathscr{L}^{\prime}(x)}{F^{\prime}(x)} \\
\mathscr{L}_{F F}(x) & =\frac{1}{F^{\prime}(x)}\left(\frac{\mathscr{L}^{\prime}}{F^{\prime}}\right)^{\prime}=\frac{\mathscr{L}^{\prime \prime} F^{\prime}-F^{\prime \prime} \mathscr{L}^{\prime}}{F^{\prime 3}}
\end{aligned}
$$

where prime' denotes differentiation with respect to $x$. If we need to obtain analytic form for $\mathscr{L}(F)$ we should substitute $x$ from (19) into (22) but that form will be complicated. Hence we plot numerical diagram for $\{F, \mathscr{L}\}$ by substituting numerical values of Tables 1 and 2 into (19) and (22) and by computing their numerical values. Diagram for $\{F, \mathscr{L}\}$ given by Figure 1 shows that effects of the NEM fields are negligible for $|q|>1$ but not for $|q|<1$. However we will need to exact form of the functions $\left\{\mathscr{L}(x), \mathscr{L}_{F}(x), \mathscr{L}_{F F}(x)\right\}$ to study location of effective metric horizons, gravitational lensing images, and their magnifications. To do so we will use numerical method as follows. One can infer that effective metric of the background (1) defined by (15) reads

$$
\begin{aligned}
d s_{\mathrm{eff}}^{2}= & -A(r) d t^{2}+B(r) d r^{2} \\
& +r^{2} C(r)\left(d \theta^{2}+\sin ^{2} \theta d \varphi^{2}\right),
\end{aligned}
$$


where we defined

$$
\begin{aligned}
& A(r)=\frac{16 H(r) \mathscr{L}_{F}}{16\left(\mathscr{L}_{F}+F \mathscr{L}_{F F}\right)^{2}-F^{2} \mathscr{L}_{F F}^{2}}, \\
& B(r)=\frac{1}{H(r)} \frac{16 \mathscr{L}_{F}}{16\left(\mathscr{L}_{F}+F \mathscr{L}_{F F}\right)^{2}-F^{2} \mathscr{L}_{F F}^{2}}, \\
& C(r)=\frac{8\left(2 \mathscr{L}_{F}+4 F \mathscr{L}_{F F}\right)}{16\left(\mathscr{L}_{F}+F \mathscr{L}_{F F}\right)^{2}-F^{2} \mathscr{L}_{F F}^{2}} .
\end{aligned}
$$

Radius of the event horizon $r_{H}$ can be obtained by solving the equation $A(r)=0$ in the presence of the NEM field and by choosing its greatest positive root. According to study of black hole gravitational lensing, photon sphere construction is one of the important characters which must be considered here. It comes from energy condition [60] and is a particular hypersurface ( $r=$ constant) which does not evolve with time. In other words any null geodesic initially tangent to the photon sphere hypersurface will remain tangent to it. It is made from circulating photons turning around the black hole center. Radius of the photon sphere $r_{p s}$ is the greatest positive solution of [48]

$$
\left(\frac{1}{r^{2}} \frac{A(r)}{C(r)}\right)_{\left.\right|_{r=r_{p s}^{\mathrm{eff}}}}^{\prime}=0 .
$$

Setting (17), (25) reads

$$
\begin{aligned}
& A(r)=H(r), \\
& B(r)=\frac{1}{H(r)}, \\
& C(r)=1
\end{aligned}
$$

which describe the original background space-time (1) without NEM field effects for which (26) reduces to the following form.

$$
\left(\frac{H(r)}{r^{2}}\right)_{\left.\right|_{r=r p s}}^{\prime}=0
$$

Diagrams of (26) and (28) are plotted for larger roots in Figure 1. Linear branch of the right panel of the diagram in Figure 1 predicts large scale photon spheres for $|q|>1$ which are formed only in presence of NEM field. This linear branch of the effective photon sphere diagram can be approximated with the following equation.

$$
x_{p s}^{\mathrm{eff}}(|q|>1) \approx 3.643|q|-0.796
$$

which rises by increasing $|q| \rightarrow \infty$. We calculated numerical values of the above photon sphere radius for $1<|q|<36$ and collected them in Table 2. Corresponding diagram is given in Figure 1. One can deduct from Figure 1 that we have small scale photon sphere for $|q|<1$ from both of the effective metric (24) and the original background (1). Hence our obtained gravitational lensing results from (1) can be compared with ones obtained from (24) only for $|q|<1$. Thus we collect numerical solutions of the both photon sphere equations (26) and (28) for $|q|<1$ in Table 1 . We will need them to evaluate numerical values of deflection angle, image locations, and corresponding magnifications. We will study gravitational lensing of the system separately for two different regimes $|q|>1$ and $|q|<1$, respectively, as follows. At first step we proceed to evaluate numerical values of the deflection angle of bending light rays.

\section{Deflection Angle}

If the light beam passes through the ABG black hole without rotation, then weak lensing occurs. In the latter case closest approach distance of the bending light rays from the black hole center $r_{0}$ become larger than the photon sphere radius and two nonrelativistic images are usually formed. They are called primary and secondary images. In general, bending angle of light rays is obtained by solving the null geodesics equation defined by (13) as follows [61].

$$
\alpha_{\text {eff }}\left(r_{0}\right)=I_{\text {eff }}\left(r_{0}\right)-\pi,
$$

where

$$
\begin{aligned}
& I_{\mathrm{eff}}\left(x_{0}\right) \\
& \quad=2 \int_{x_{0}>x_{p s}}^{\infty} \frac{\sqrt{A(x) B(x) / C^{2}(x)}}{\sqrt{A\left(x_{0}\right) / x_{0}^{2} C\left(x_{0}\right)-A(x) / x^{2} C(x)}} \frac{d x}{x^{2}} .
\end{aligned}
$$

Inserting

$$
z=\frac{x_{0}}{x}
$$

the integral equations (31) become

$$
I_{\text {eff }}\left(x_{0}\right)=2 \int_{0}^{1} \frac{\Gamma\left(x_{0} / z\right)}{\sqrt{\Omega\left(x_{0}\right)-\Omega\left(x_{0} / z\right) z^{2}}} d z,
$$

where we defined

$$
\begin{aligned}
\Gamma\left(\frac{x_{0}}{z}\right) & =\Omega \sqrt{\frac{B}{A}}=\frac{\mathscr{L}_{F}}{\mathscr{L}_{F}+2 F \mathscr{L}_{F F}}, \\
\Omega\left(\frac{x_{0}}{z}\right) & =\frac{A}{C}=H \Gamma .
\end{aligned}
$$

According to method given in [59], we calculate Taylor series expansion of the functions $\Gamma\left(x_{0} / z\right)$ and $\Omega\left(x_{0}\right)-\Omega\left(x_{0} / z\right) z^{2}$ versus powers of $(1-z)$ as follows.

$$
\begin{aligned}
\Gamma\left(\frac{x_{0}}{z}\right)= & \Gamma_{0}+\Gamma_{1}(1-z)+\Gamma_{2}(1-z)^{2} \\
& +O(3), \\
\Omega\left(x_{0}\right)-\Omega\left(\frac{x_{0}}{z}\right) z^{2}= & \Omega_{1}(1-z)+\Omega_{2}(1-z)^{2} \\
& +O(3),
\end{aligned}
$$


TABLE 2: Solutions of the effective photon sphere equation (29) for $|q|>1$.

\begin{tabular}{|c|c|c|c|c|c|}
\hline$|q|, x_{p s}^{\mathrm{eff}}$ & $|q|, x_{p s}^{\mathrm{eff}}$ & $|q|, x_{p s}^{\mathrm{eff}}$ & $|q|, x_{p s}^{\mathrm{eff}}$ & $|q|, x_{p s}^{\mathrm{eff}}$ & $|q|, x_{p s}^{\mathrm{eff}}$ \\
\hline $1,2.847$ & $7,24.705$ & $13,46.563$ & $19,68.421$ & $25,90.279$ & $31,112.137$ \\
\hline $2,6.490$ & $8,28.348$ & $14,50.206$ & $20,72.064$ & $26,93.922$ & $32,115.780$ \\
\hline $3,10.133$ & $9,31.991$ & $15,53.849$ & $21,75.707$ & $27,97.565$ & $33,119.423$ \\
\hline $4,13.776$ & $10,35.634$ & $16,57.492$ & $22,79.350$ & $28,101.208$ & $34,123.066$ \\
\hline $5,17.419$ & $11,39.277$ & $17,61.135$ & $23,82.993$ & $29,104.851$ & $35,126.709$ \\
\hline $6,21.062$ & $12,42.920$ & $18,64.778$ & $24,86.636$ & $30,108.494$ & $36,130.352$ \\
\hline
\end{tabular}

where we defined

$$
\begin{aligned}
\Gamma_{0} & =\Gamma\left(x_{0}\right), \\
\Gamma_{1} & =x_{0} \Gamma^{\prime}\left(x_{0}\right), \\
\Gamma_{2} & =x_{0} \Gamma^{\prime}\left(x_{0}\right)+\frac{x_{0}^{2} \Gamma^{\prime \prime}\left(x_{0}\right)}{2}, \\
\Omega_{1} & =2 \Omega\left(x_{0}\right)-x_{0} \Omega^{\prime}\left(x_{0}\right), \\
\Omega_{2} & =x_{0} \Omega^{\prime}\left(x_{0}\right)-\Omega\left(x_{0}\right)-\frac{x_{0}^{2} \Omega^{\prime \prime}\left(x_{0}\right)}{2}
\end{aligned}
$$

in which prime ${ }^{\prime}$ denotes differentiation with respect to its argument $x$. Inserting (35) and neglecting their higher order terms, the integral equation (33) becomes

$$
I_{\mathrm{eff}}\left(x_{0}\right) \approx 2 \int_{0}^{1} d z\left[\frac{\Gamma_{0}+\Gamma_{1}(1-z)+\Gamma_{2}(1-z)^{2}}{\sqrt{\Omega_{1}(1-z)+\Omega_{2}(1-z)^{2}}}\right]
$$

where its solution reads

$$
\begin{aligned}
& I_{\text {eff }}\left(x_{0}\right)=\frac{1}{\sqrt{\Omega_{2}\left(x_{0}\right)}} \sqrt{1+\frac{\Omega_{1}\left(x_{0}\right)}{\Omega_{2}\left(x_{0}\right)}}\left[2 \Gamma_{1}\left(x_{0}\right)\right. \\
& \left.+\Gamma_{2}\left(x_{0}\right)-\frac{3}{2} \Gamma_{2}\left(x_{0}\right) \frac{\Omega_{1}\left(x_{0}\right)}{\Omega_{2}\left(x_{0}\right)}\right] \\
& -\frac{1}{\sqrt{\Omega_{2}\left(x_{0}\right)}}\left[2 \Gamma_{0}\left(x_{0}\right)-\Gamma_{1}\left(x_{0}\right) \frac{\Omega_{1}\left(x_{0}\right)}{\Omega_{2}\left(x_{0}\right)}\right. \\
& \left.+\frac{3}{4} \Gamma_{2}\left(x_{0}\right)\left(\frac{\Omega_{1}\left(x_{0}\right)}{\Omega_{2}\left(x_{0}\right)}\right)^{2}\right] \\
& \quad \times \ln \left[1+2 \mathrm{frac}\left(1-\sqrt{1+\frac{\Omega_{1}\left(x_{0}\right)}{\Omega_{2}\left(x_{0}\right)}}\right) \frac{\Omega_{1}\left(x_{0}\right)}{\Omega_{2}\left(x_{0}\right)}\right] .
\end{aligned}
$$

WDL and SDL of bending light rays are regimes where $I_{\text {eff }}\left(x_{0}\right) \nrightarrow \infty$ and $I_{\text {eff }}\left(x_{0}\right) \rightarrow \infty$, respectively. This restricts us to choose particular regimes of the ratio $\Omega_{1} / \Omega_{2}$ given by (39). Substituting (34) and (37) into the photon sphere equation (26) and setting $x_{0}=x_{p s}^{\text {eff }}$ one can get $x_{p s} \Omega^{\prime}\left(x_{p s}\right)-2 \Omega\left(x_{p s}\right)=$ $\Omega_{1}\left(x_{p s}\right)=0$. The latter condition is valid for moving light rays near the photon sphere for which $I_{\text {eff }} \rightarrow \infty$. In other words one infers $\Omega_{1}\left(x_{0} \neq x_{p s}^{\text {eff }}\right) \neq 0$ for WDL. Hence we can use asymptotic expansion of the solution (39) for $x_{0}>x_{p s}$ and $\forall q$ as follows.

4.1. Weak Lensing Deflection Angles. One can obtain asymptotic expansion series of the functions $\Omega_{1,2}\left(x_{0}\right)$ and $\Gamma_{0,1,2}\left(x_{0}\right)$ which up to terms in order of $O\left(x_{0}^{-3}\right)$ become, respectively,

$$
\begin{aligned}
& \Omega_{1}\left(x_{0}\right) \approx 2-\frac{3 / 8}{x_{0}}-\frac{\left(32 q^{2}+345 / 8\right)}{x_{0}^{2}} \\
& +\frac{\left(4305 q^{2} / 16+25875 / 128\right)}{x_{0}^{3}}, \\
& \Omega_{2}\left(x_{0}\right) \approx-1+\frac{3 / 8}{x_{0}}+\frac{\left(1035 / 16+48 q^{2}\right)}{x_{0}^{2}} \\
& -\frac{\left(4305 q^{2} / 8+25875 / 64\right)}{x_{0}^{3}} \\
& \Gamma_{0}\left(x_{0}\right) \approx 1+\frac{15 / 8}{x_{0}}-\frac{\left(9 q^{2}+225 / 32\right)}{x_{0}^{2}} \\
& +\frac{\left(495 q^{2} / 16+3375 / 128\right)}{x_{0}^{3}} \\
& \Gamma_{1}\left(x_{0}\right) \approx-\frac{15 / 8}{x_{0}}+\frac{\left(18 q^{2}+225 / 16\right)}{x_{0}^{2}} \\
& -\frac{\left(1485 q^{2} / 16+10125 / 128\right)}{x_{0}^{3}} \\
& \Gamma_{2}\left(x_{0}\right) \approx-\frac{\left(9 q^{2}+225 / 32\right)}{x_{0}^{2}} \\
& +\frac{\left(1485 q^{2} / 16+10125 / 128\right)}{x_{0}^{3}} \text {. }
\end{aligned}
$$

Substituting (40) into the integral solution (39) and using some simple calculations, one can infer

$$
\begin{aligned}
& I_{\mathrm{eff}}^{\text {weak }}\left(x_{0}>x_{p s}^{\mathrm{eff}}\right) \approx \pi-\frac{\left(33 / 8+471 / 128+9 \pi q^{2} / 2\right)}{x_{0}} \\
& +\frac{\left(741 \pi q^{2} / 32+64863 \pi / 2048-8841 q / 1024-997 q^{2} / 16\right)}{x_{0}^{3}} .
\end{aligned}
$$



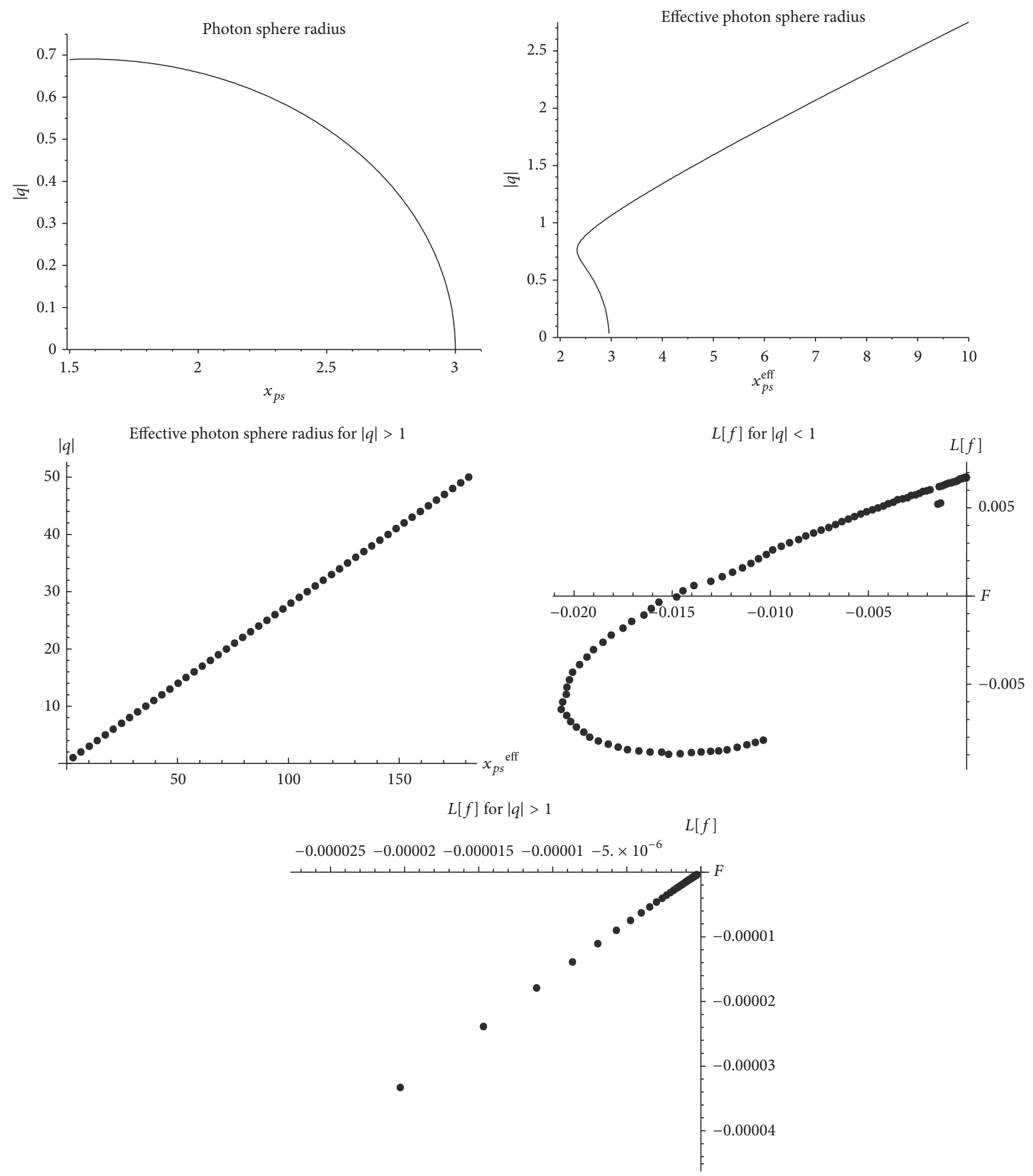

FIGURE 1: Diagrams of photon sphere locations $x_{p s}$ and NEM field Lagrangian density $L[F]$.

Defining

$$
y=\frac{x_{0}}{x_{p s}^{\mathrm{eff}}}>1
$$

and inserting (41) the deflection angle (30) becomes

$$
\alpha_{\mathrm{eff}}^{\text {weak }}\left(y_{0}>1\right) \approx-\frac{M}{y_{0}}+\frac{N}{y_{0}^{3}}
$$

for WDL where we defined

$$
\begin{aligned}
& M\left(x_{p s}^{\mathrm{eff}}, q\right)=\frac{1}{x_{p s}^{\mathrm{eff}}}\left(\frac{33}{8}+\frac{471}{128}+\frac{9 \pi q^{2}}{2}\right), \\
& N\left(x_{p s}^{\mathrm{eff}}, q\right)
\end{aligned}
$$




$$
=\frac{1}{\left(x_{p s}^{\mathrm{eff}}\right)^{3}}\left(\frac{741 \pi q^{2}}{32}+\frac{64863 \pi}{2048}-\frac{8841 q}{1024}-\frac{997 q^{2}}{16}\right) .
$$

Applying (17) and (34) we obtain

$$
\begin{aligned}
\Omega\left(x_{0}\right) & =H, \\
\Omega_{1}\left(x_{0}\right) & =2 H-x_{0} H^{\prime}, \\
\Omega_{2}\left(x_{0}\right) & =x_{0} H^{\prime}-H-\frac{x_{0}^{2} H^{\prime \prime}}{2}, \\
\Gamma(x) & =\Gamma_{0}(x)=1, \\
\Gamma_{1}(x) & =0=\Gamma_{2}(x)
\end{aligned}
$$

which are applicable for WDL in absence of the NEM field effects. In the latter case asymptotic behavior of the functions given by (45) for $x_{0}>x_{p s}$ reads

$$
\begin{aligned}
& \Omega_{1}\left(x_{0}\right) \approx 2-\frac{6}{x_{0}}+\frac{4 q^{2}}{x_{0}^{2}}+\frac{15 q^{2}}{x_{0}^{3}}, \\
& \Omega_{2}\left(x_{0}\right) \approx-1+\frac{6}{x_{0}}-\frac{6 q^{2}}{x_{0}^{2}}-\frac{30 q^{2}}{x_{0}^{3}} .
\end{aligned}
$$

Substituting (45) and (46) asymptotic series expansion of the solution (39) is obtained as follows.

$$
\begin{aligned}
I^{\text {weak }}\left(x_{0}\right) \approx & \pi+\frac{3(\pi-2)}{x_{0}} \\
& +\frac{\left(8 q^{2}-36-6 \pi q^{2}+27 \pi / 2\right)}{x_{0}^{2}} \\
& +\frac{\left(123 q^{2}-198-42 q^{2} \pi+135 \pi / 2\right)}{x_{0}^{3}}
\end{aligned}
$$

where by substituting it into (30) one can obtain weak deflection angle (WDA) of bending light rays in absence of the NEM field such that

$$
\alpha^{\text {weak }}\left(y_{0}>1\right) \approx \frac{S}{y_{0}}+\frac{R}{y_{0}^{2}}+\frac{Q}{y_{0}^{3}},
$$

where we defined

$$
\begin{aligned}
& S\left(x_{p s}, q\right)=\frac{3(\pi-2)}{x_{p s}}, \\
& R\left(x_{p s}, q\right)=\frac{\left(8 q^{2}-36-6 \pi q^{2}+27 \pi / 2\right)}{x_{p s}^{2}}, \\
& Q\left(x_{p s}, q\right)=\frac{\left(123 q^{2}-198-42 q^{2} \pi+135 \pi / 2\right)}{x_{p s}^{3}} .
\end{aligned}
$$

Diagrams of (43) and (48) are plotted in Figure 2 for ansatz $y_{0}=10$ by inserting numerical values given in Table 1 . It is suitable to obtain the following averaged deflection angles.

$$
\begin{aligned}
& \sigma_{\mathrm{eff}}^{\text {weak }}=\bar{\alpha}_{\mathrm{eff}}^{\text {weak }} \approx-\frac{\bar{M}}{y_{0}}+\frac{\bar{N}}{y_{0}^{3}}, \\
& \sigma^{\text {weak }}=\bar{\alpha}^{\text {weak }} \approx \frac{\bar{S}}{y_{0}}+\frac{\bar{R}}{y_{0}^{2}}+\frac{\bar{Q}}{y_{0}^{3}},
\end{aligned}
$$

where we defined all mean coefficients

$$
\overline{\mathscr{C}}=\frac{1}{N} \sum_{i=1}^{N} \mathscr{C}\left(x_{p s_{i}}, q_{i}\right)
$$

in which $\mathscr{C}=\{M, N, S, R, Q\}$. Substituting numerical values of Table 1 we obtain

$$
\begin{aligned}
& \bar{M}=4.79, \\
& \bar{N}=5.41, \\
& \bar{S}=1.32, \\
& \bar{R}=0.64, \\
& \bar{Q}=0.80
\end{aligned}
$$

for which (50) and (51) become, respectively,

$$
\begin{aligned}
& \sigma_{\text {eff }}^{\text {weak }}\left(y_{0}>1\right) \approx-\frac{4.79}{y_{0}}+\frac{5.41}{y_{0}^{3}}, \\
& \sigma^{\text {weak }}\left(y_{0}>1\right) \approx \frac{1.32}{y_{0}}+\frac{0.64}{y_{0}^{2}}+\frac{0.80}{y_{0}^{3}} .
\end{aligned}
$$

Diagrams of the above equations are given in Figure 2. They show that the sign of deflection angle is changed in presence of the NEM fields with respect to its sign without effects of the NEM field.

4.2. Strong Lensing Deflection Angles. In case of SDL we write Taylor series expansion of the solution (39) at neighborhood of $x_{0} \rightarrow x_{p s}^{\text {eff }}$ or equivalently $y_{0} \rightarrow 1$. In the latter case we must be obtain Taylor series expansion of the functions $\Omega_{1,2}\left(x_{0}\right)$ and $\Gamma_{0,1,2}\left(x_{0}\right)$ which up to terms in order of $O(3)$ become, respectively,

$$
\begin{aligned}
\Omega_{1}\left(x_{0}\right) \approx & P_{p s}\left(y_{0}-1\right)+Q_{p s}\left(y_{0}-1\right)^{2}, \\
\Omega_{2}\left(x_{0}\right) \approx & \Omega_{2}\left(x_{p s}\right)+R_{p s}\left(y_{0}-1\right)-Q_{p s}\left(y_{0}-1\right)^{2}, \\
\Gamma_{0}\left(x_{0}\right) \approx & \Gamma_{0}\left(x_{p s}\right)+U_{p s}\left(y_{0}-1\right)+V_{p s}\left(y_{0}-1\right)^{2}, \\
\Gamma_{1}\left(x_{0}\right) \approx & U_{p s}+\left(U_{p s}+2 V_{p s}\right)\left(y_{0}-1\right) \\
& +\left(2 V_{p s}+W_{p s}\right)\left(y_{0}-1\right)^{2}, \\
\Gamma_{2}\left(x_{0}\right) \approx & U_{p s}+V_{p s}+\left(U_{p s}+4 V_{p s}+W_{p s}\right)\left(y_{0}-1\right) \\
& +3\left(V_{p s}+W_{p s}\right)\left(y_{0}-1\right)^{2},
\end{aligned}
$$



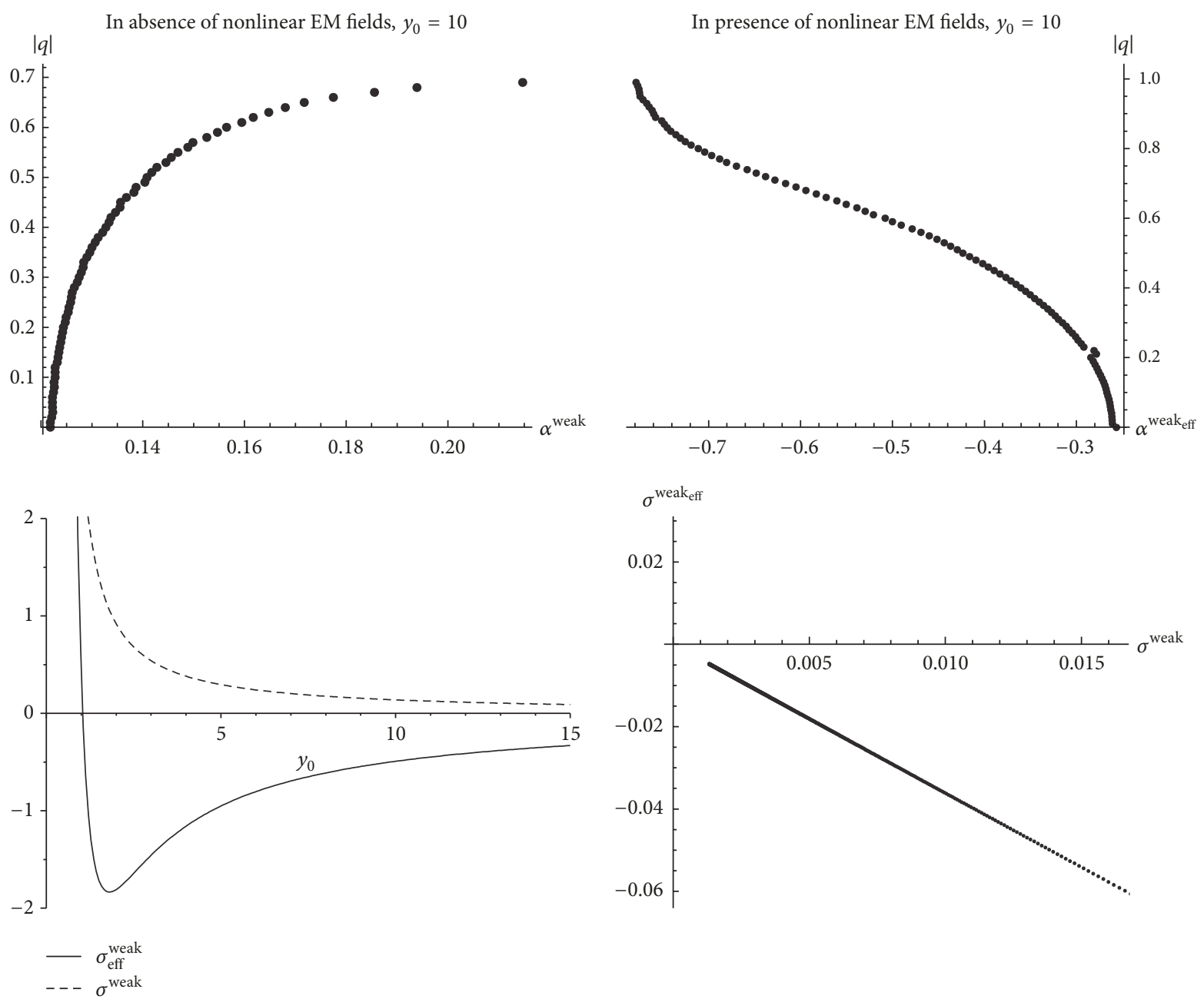

FIGURE 2: Diagrams of weak lensing deflection angles for $|q|<1$.

where we defined

$$
\begin{aligned}
P_{p s} & =2 \Omega_{2}\left(x_{p s}\right) \\
\Omega_{2}\left(x_{p s}\right) & =\Omega\left(x_{p s}\right)-\frac{x_{p s}^{2} \Omega^{\prime \prime}\left(x_{p s}\right)}{2} \\
R_{p s} & =-x_{p s}^{2} \Omega^{\prime \prime}\left(x_{p s}\right) \\
Q\left(x_{p s}\right) & =-\frac{x_{p s}^{3} \Omega^{\prime \prime \prime}\left(x_{p s}\right)}{2} \\
U_{p s} & =x_{p s} \Gamma_{0}^{\prime}\left(x_{p s}\right) \\
V_{p s} & =\frac{x_{p s}^{2} \Gamma_{0}^{\prime \prime}\left(x_{p s}\right)}{2} \\
W_{p s} & =\frac{x_{p s^{3}} \Gamma_{0}^{\prime \prime \prime}\left(x_{p s}\right)}{2} .
\end{aligned}
$$

Inserting (56) into the solution (39) one can obtain SDL of bending angle (30) as follows.

$$
\alpha_{\mathrm{eff}}^{\text {strong }}\left(y_{0} \rightarrow 1\right) \approx b+a \ln \left(y_{0}-1\right),
$$

where we defined

$$
\begin{aligned}
& b=-\pi+\frac{3 U_{p s}+2 \ln 2 V_{p s} \Gamma_{0}\left(x_{p s}\right)}{\sqrt{\Omega_{2}\left(x_{p s}\right)}}, \\
& a=-\frac{2 \Gamma_{0}\left(x_{p s}\right)}{\sqrt{\Omega_{2}\left(x_{p s}\right)}} .
\end{aligned}
$$

Divergency of (58) for $y_{0}=1$ can be described by Bozza formalism as follows.

$$
\alpha_{\mathrm{eff}}^{\text {strong }}=\alpha_{n}=\Delta \alpha_{n}+2 n \pi,
$$

where $n=0, \pm 1, \pm 2, \pm 3, \ldots$, means $n$th circulation of light rays around center of the lens to make $n$th relativistic image with deflection angle $\alpha_{n}$ where differences between these angles should satisfy the condition $0<\Delta \alpha_{n}<1$. Nonrelativistic images are determined by setting $n=0$ and relativistic images with positive (negative) parity are determined by setting $n=1,2, \ldots(n=-1,-2, \ldots)$ where one can obtain $\Delta \alpha_{-n}=2 \alpha-\Delta \alpha_{n}$. In case of retrolensing where observer is located between source and lens, the light 
rays come back after turning around the lens (see Figure 1 at $[46,47])$. In the latter case the parameter $2 n$ given in the formula (60) must be replaced with $2 n-1$. In case of SDL in absence of NEM fields we should use (58) but by inserting

$$
\begin{aligned}
P_{p s} & =2 H\left(x_{p s}\right)-x_{p s}^{2} H^{\prime \prime}\left(x_{p s}\right) \\
U_{p s} & =-\frac{2 x_{p s} H^{\prime}}{H^{3}} \\
V_{p s} & =-\frac{x_{p s}^{2} H^{\prime \prime}}{H^{3}}+\frac{3 x_{p s}^{2} H^{\prime 2}}{H^{4}} \\
R_{p s} & =-x_{p s}^{2} H^{\prime \prime} \\
Q_{p s} & =-\frac{x_{p s}^{3} H^{\prime \prime \prime}}{2}
\end{aligned}
$$

Now we study image locations for WDL and SDL of gravitational lensing.

\section{Images Locations}

In order to calculate location of the images in WDL, we choose Ohanian lens equation [54] which has high accuracy and so lower errors with respect to other lens equations [62]. It has the advantage of being the closest relative of the exact lens equation, since it contains just the asymptotic approximation without any additional assumption. It can be rewritten versus observational coordinates, namely, the image position $\theta$, the source position $\beta$, and the deflection angle of bending light rays $\alpha^{\text {weak }}$ as follows (see [62] for more discussions).

$$
\arcsin \left(D_{L} \sin \theta\right)-\arcsin \left(D_{S} \sin \beta\right)=\alpha-\theta
$$

in which we defined

$$
\begin{aligned}
& D_{L}=\frac{d_{O L}}{d_{L S}}, \\
& D_{S}=\frac{d_{O S}}{d_{L S}} .
\end{aligned}
$$

In the above equations $d_{O S}$ is distance between the observer and the source, $d_{\mathrm{OL}}$ is distance between the observer and the lens, and $d_{L S}$ is distance between the lens and the source. $\theta$ is formed when a line passing through the observer and the image crosses the optical axis (line passing through the observer and the lens). $\beta$ is formed when a line passing through the observer and the source crosses the optical axis. One can obtain general solutions of the lens equation (62) as follows.

$$
\begin{aligned}
& \theta_{K}(\alpha, \beta) \\
& =\arctan \left[\frac{D_{S} \cos \alpha \sin \beta+K \sin \alpha \sqrt{1-D_{S}^{2} \sin ^{2} \beta}}{D_{L}-D_{S} \sin \alpha \sin \beta+\cos \alpha \sqrt{1-D_{S}^{2} \sin ^{2} \beta}}\right],
\end{aligned}
$$

where $K= \pm 1$. It has some real solutions for

$$
\sin \beta \leq \frac{1}{D_{S}} .
$$

In the following we use (64) to obtain locations of nonrelativistic and relativistic image.

5.1. Weak Lensing Images. For WDL with large distances between the lens, the source, and the observer located in a straight line, one can infer [62]

$$
D_{S} \approx D_{L}+1 \text {, }
$$

where $D_{L} \gg 1$ must be substituted via experimental date. As a realistic example of gravitational lens we consider a big black hole located in the center of galaxy and study image locations of a star located far from it. This black hole is called Sgr A* $[48,55-57]$. Its mass is estimated as $3.6 \times 10^{6} \mathrm{M}_{\odot}$ and its distance from the earth is $d_{\mathrm{OL}}=8 \mathrm{kpc}=2.47 \times 10^{17} \mathrm{~m}$ with corresponding Schwarzschild radius $R_{S C H}=10^{10} \mathrm{~m}$. We consider a source to be a star located at distance $d_{L S}=$ $1.7 \times 10^{13} \mathrm{~m}$ from the black hole which is far from the margin of the accretion disk of the black hole, so it may not be falling toward the black hole center. For the latter black hole we will have

$$
D_{L} \approx 1.45 \times 10^{4} \approx D_{S} .
$$

The relations (65) and (67) lead us to choose

$$
\sin \beta \approx \beta \leq \beta_{M},
$$

for WDL of gravitational lensing where we defined

$$
\beta_{M}=\frac{1}{D_{S}} \approx \frac{1}{D_{L}} \approx 7 \times 10^{-5} \operatorname{Rad} \equiv 40107 \mu \operatorname{arcsec}
$$

in which the subscript $M$ denotes the word "Maximum." For critical source $\beta=\beta_{M}$ the lens equation (64) reads

$$
\theta_{M}(\alpha)=\arctan \left[\frac{\cos \alpha}{D_{L}-\sin \alpha}\right]
$$

which for WDL $\alpha \rightarrow 0$ leads to the following approximation.

$$
\theta_{M} \approx \frac{1}{D_{L}} \approx 40107 \mu \operatorname{arcsec} .
$$

Defining normalized angular locations for the images and the source as

$$
\begin{aligned}
& \theta^{*}=\frac{\theta}{\theta_{M}}, \\
& \beta^{*}=\frac{\beta}{\beta_{M}}
\end{aligned}
$$

we can obtain Taylor series expansion of the lens equation (64) at neighborhood of $\left(|\alpha|,\left|\beta^{*}\right|\right) \ll(1,1)$ as follows.

$$
\begin{aligned}
\theta_{K}^{*} \approx & \frac{K D_{L}}{1+D_{L}} \alpha+\frac{D_{L}}{1+D_{L}} \beta^{*}-\frac{K}{6} \frac{D_{L}^{2}\left(D_{L}-1\right)}{\left(1+D_{L}\right)^{3}} \alpha^{3} \\
& -\frac{D_{L}}{2} \frac{D_{L}^{2}-2 K D_{L}+2+D_{L}-2 K}{\left(1+D_{L}\right)^{3}} \beta^{*} \alpha^{2}
\end{aligned}
$$




$$
\begin{aligned}
& -\frac{D_{L}}{2} \frac{\left(D_{L}^{2} K+D_{L} K-2 D_{L}+2 K-2\right)}{\left(1+D_{L}\right)^{3}} \alpha \beta^{* 2} \\
& +\frac{D_{L}}{6} \frac{1+3 D_{L}}{\left(1+D_{L}\right)^{3}} \beta^{* 3} \\
& +\frac{K D_{L}}{120} \frac{\left(D_{L}^{4}+11 D_{L}^{2}-11 D_{L}^{3}-D_{L}\right)}{\left(1+D_{L}\right)^{5}} \alpha^{5}+\cdots
\end{aligned}
$$

which in limits $D_{L} \rightarrow \infty$ become

$$
\begin{aligned}
\theta_{K}^{*} \approx & K \alpha+\beta^{*}-\frac{\alpha^{2} \beta^{*}}{2}-\frac{K \alpha \beta^{* 2}}{2}-\frac{K}{6} \alpha^{3}+\frac{K}{120} \alpha^{5} \\
& +\cdots
\end{aligned}
$$

This is primary image location and by transforming $\beta^{*} \rightarrow$ $-\beta^{*}$ as $\theta_{K}^{*}\left(-\beta^{*}\right)$ one can obtain secondary image location for which $K=+1(-1)$ describes right (left) handed bending angles of light rays. Setting $K=+1, \beta^{*}=1$, and $y_{0}=$ 10 and computing numerical values of the deflection angles (43) and (48) via numerical values of Table 1 (see Figure 2) we plot numerical diagrams for $\theta_{i}^{\text {eff }}$ and $\theta_{i}$ against different values $\left|q_{i}\right|$ in Figure 3. Also we substitute (54) into the weak lens equation (74) by setting $K=+1$ and plot mean weak image locations in Figure 3 versus $|q|$ in Figure 3. Now we are in a position to obtain location of relativistic image in the subsequent section.

5.2. Strong Lensing Images. The Virbhadra-Ellis lens equation given by [63]

$$
\tan \beta=\tan \theta-D[\tan (\alpha-\theta)+\tan \theta]
$$

is useful to study gravitational lensing in SDL. In the above lens equation we have $D=d_{l s} / d_{\text {os }}$ for standard lensing in which the lens is located between the observer and the source, $D=d_{o s} / d_{o l}$ for situations where the source is located between the observer and the lens, and $D=d_{o s} / d_{l s}$ for situations where the observer is located between the source and the lens (the retrolensing). The lensing effects are more important when the objects are highly aligned, in which $\beta, \theta$ are small and $\alpha$ is close to $2 n \pi+\Delta \alpha_{n}$ for the standard lensing and $(2 n+1) \pi+\Delta \alpha_{n}$ for the retrolensing with $0<\Delta \alpha_{n} \ll 1$, $n=1,2, \ldots$ In the latter case we can use the approximations $\tan \theta \approx \theta$ and $\tan \beta \approx \beta$ for the lens equation (75) and substitute $\alpha_{n}=2 n \pi+\Delta \alpha_{n}$ such that (see (32) in [59])

$$
\beta=\theta-D \Delta \alpha_{n} \text {. }
$$

Defining coordinate independent impact parameter

$$
u=\frac{r}{\sqrt{\Omega(r)}},
$$

one can obtain its Taylor series expansion by applying (56)(57) as follows (see (28) in [59])

$$
y_{0}-1 \approx \sqrt{2}\left(\frac{\Omega_{2}\left(x_{p s}\right)}{\Omega\left(x_{p s}\right)}\right)^{-1 / 2}\left(\frac{u_{0}}{u_{p s}}-1\right)^{1 / 2}
$$

in which $u_{0}=d_{L O} \sin \theta$ for small $\theta$ reduces to $u_{0} \approx d_{L O} \theta$. Inserting the latter relation and (78), the strong deflection angle (58) becomes

$$
\alpha(\theta)=c_{2}-c_{1} \ln \left(\frac{d_{\mathrm{OL}}}{u_{p s}} \theta-1\right),
$$

where we defined

$$
\begin{aligned}
& c_{1}=-\frac{a}{2}, \\
& c_{2}=b+\frac{a}{2} \ln \left(\frac{2 \Omega\left(x_{p s}\right)}{\Omega_{2}\left(x_{p s}\right)}\right) .
\end{aligned}
$$

One can obtain $\theta(\alpha)$ by inverting (79) as

$$
\theta(\alpha)=\frac{u_{p s}}{d_{O L}}\left(1+e^{\left(c_{2}-\alpha\right) / c_{1}}\right)
$$

and by substituting (60) the above equation can be rewritten as

$$
\theta_{n}=\frac{u_{p s}}{d_{O L}}\left(1+e^{\left(c_{2}-2 n \pi-\Delta \alpha_{n}\right) / c_{1}}\right) .
$$

By making first order Taylor series expansion of the above equation around $\alpha=2 n \pi$ the angular position of $n$th relativistic image is obtained as

$$
\theta_{n} \approx \theta_{n}^{(0)}-\zeta_{n} \Delta \alpha_{n}
$$

where we defined

$$
\begin{aligned}
\theta_{n}^{(0)} & =\frac{u_{p s}}{d_{O L}}\left[1+e^{\left(c_{2}-2 n \pi\right) / c_{1}}\right], \\
\zeta_{n} & =\frac{u_{p s}}{c_{1} d_{O L}} e^{\left(c_{2}-2 n \pi\right) / c_{1}} .
\end{aligned}
$$

Eliminating $\Delta \alpha_{n}$ between (76) and (83) we obtain

$$
\theta_{n}=\left(1+\frac{\zeta_{n}}{D}\right)^{-1}\left(\theta_{n}^{(0)}+\frac{\zeta_{n}}{D} \beta\right)
$$

in which $0<\zeta_{n} / D \ll 1$ and so we can use approximation $\left(1+\zeta_{n} / D\right)^{-1} \approx 1-\zeta_{n} / D$. In the latter case the equation (86) reads

$$
\theta_{n} \approx \theta_{n}^{(0)}+\frac{\zeta_{n}}{D}\left(\beta-\theta_{n}^{(0)}\right)
$$

The second term in the above lens equation is more smaller than the first term which means all relativistic image locations lie very close to $\theta_{n}^{(0)}$. There are other sets of relativistic images by changing $\theta_{n}^{(0)} \rightarrow-\theta_{n}^{(0)}$ into the above lens equation. In case of perfect aligned $\beta=0$ the above lens equation reaches to

$$
\theta_{n}^{E}=\left(1-\frac{\zeta_{n}}{D}\right) \theta_{n}^{(0)}
$$

which describes angular location of $n$th relativistic Einstein ring. 



FIGURE 3: Diagrams of weak lensing primary image locations for $|q|<1$.

5.2.1. Magnifications. The magnification $\mu$ of an image is defined as the ratio of flux of the image to flux of unlensed source. It has two components called tangential $\mu_{t}=$ $\sin \theta / \sin \beta$ and radial $\mu_{r}=d \theta / d \beta$ and their multiplication makes the magnification

$$
\mu=\left|\frac{\sin \beta}{\sin \theta} \frac{d \beta}{d \theta}\right|^{-1} .
$$

The above equation denotes primary image $\theta^{p}(\beta)$ magnifications with positive parity. Inserting the secondary image location $\theta^{s}(\beta)=\theta^{P}(-\beta)$ into the magnification equation (89) one can obtain secondary image magnification $\mu_{\text {weak }}^{s}(\beta)=$ $\mu_{\text {weak }}^{p}(-\beta)$ with negative parity. In the microlensing state two images in WDL are not resolved and so the main observables that should be considered are the total magnification $\mu_{\text {tot }}$ and magnification-weighted-centroid $\mu_{\text {cent }}$ defined by, respectively, [64]

$$
\begin{aligned}
& \mu_{\mathrm{tot}}=\left|\mu_{s}\right|+\left|\mu_{p}\right|, \\
& \mu_{\text {cent }}=\frac{\theta_{p}\left|\mu_{p}\right|+\theta_{s}\left|\mu_{s}\right|}{\left|\mu_{p}\right|+\left|\mu_{\mathrm{s}}\right|}
\end{aligned}
$$

and we calculate them for WDL and SDL in the next section. 
5.3. Weak Lensing Magnifications. In case of WDL we can use approximations $\sin \theta \approx \theta$ and $\sin \beta \approx \beta$ to evaluate (89) as follows.

$$
\mu^{\text {weak }} \approx\left|\frac{\beta}{\theta} \frac{d \beta}{d \theta}\right|^{-1}
$$

which by substituting (74) reads

$$
\begin{aligned}
\mu_{K}^{\text {weak }} \approx & \left|K \alpha+\beta^{*}-\frac{\alpha^{2} \beta^{*}}{2}-\frac{K \alpha \beta^{* 2}}{2}-\frac{K \alpha^{3}}{6}+\frac{K \alpha^{5}}{120}\right| \\
& \times\left|\frac{1}{\beta^{*}}-\frac{\alpha^{2}}{2 \beta^{*}}-K \alpha\right| .
\end{aligned}
$$

Inserting numerical values given in Table 2 we calculate numerical values for $\mu, \mu_{\text {tot }}$, and $\mu_{\text {cent }}$ and plot their diagrams versus $\beta^{*}$ in Figure 4 . Diagrams show that $\mu$ decreases by increasing $|q|$ in presence and absence of NEM fields. $\mu_{\text {tot }}$ has minimum value for $\beta>0(\beta<0)$ in presence (absence) of NEM fields, while corresponding $\mu_{\text {cent }}$ take $s$ on a maximum value. Furthermore we obtain from the Figure 4 that magnification of the Einstein rings is major in presence of NEM fields with respect to situations where the NEM field effect is negligible. Now we proceeded to study magnifications of images for SDL.

5.3.1. Strong Lensing Magnifications. To calculate $n$th relativistic images magnification we should determine

$$
\mu_{n} \approx\left|\frac{\beta}{\theta_{n}} \frac{d \beta}{d \theta_{n}}\right|^{-1}
$$

which by substituting (87) reads

$$
\mu_{n} \approx \frac{\zeta_{n}}{D} \frac{\theta_{n}^{(0)}}{\beta}
$$

The above magnification is valid for a point source and for extended source there is obtained a different form of the magnification (see for instance (46) in [59]). Equation (94) shows that the first relativistic image is brightest one, and the magnifications decreases exponentially by raising " $n$ ". Magnifications for retrolensing are obtained by (94) but by changing $2 n \rightarrow 2 n-1$. Taking into account both sets of relativistic images, the total magnification is defined by $\mu_{\text {tot }}^{\text {strong }}=2 \sum_{n=1}^{\infty} \mu_{n}$. Using the formula $t /(1-t)=\sum_{n=1}^{\infty} t^{n}$ and inserting (84), (85) and (94) we obtain

$$
\mu_{\text {tot }}^{\text {strong }}=\frac{1}{\beta}\left(\frac{2 u_{p s}^{2} e^{c_{2} / c_{1}}}{D c_{1} d_{\mathrm{OL}}^{2}}\right)\left[\frac{1+e^{c_{2} / c_{1}}+e^{2 \pi / c_{1}}}{e^{4 \pi / c_{1}}-1}\right]
$$

for a point source where

$$
\begin{gathered}
u_{p s}\left(x_{p s}\right)=\frac{x_{p s}}{\sqrt{\Omega\left(x_{p s}\right)}}, \\
c_{1}\left(x_{p s}\right)=\frac{\Gamma_{0}\left(x_{p s}\right)}{\sqrt{\Omega_{2}\left(x_{p s}\right)}},
\end{gathered}
$$

$$
\begin{aligned}
c_{2}\left(x_{p s}\right)= & -\pi+\frac{3 U_{p s}+V_{p s}+2 \ln 2 \Gamma_{0}\left(x_{p s}\right)}{\sqrt{\Omega_{2}\left(x_{p s}\right)}} \\
& -\frac{\Gamma_{0}\left(x_{p s}\right)}{\sqrt{\Omega_{2}\left(x_{p s}\right)}} \ln \left[\frac{2 \Omega\left(x_{p s}\right)}{\Omega_{2}\left(x_{p s}\right)}\right] .
\end{aligned}
$$

Equations (97) are obtained by substituting (59) into the relations (80).

As an example we obtain the lensing observable defined by Bozza [43] as

$$
\begin{aligned}
& s=\theta_{1}-\theta_{\infty}, \\
& r=\frac{\mu_{1}}{\sum_{n=2}^{\infty} \mu_{n}}
\end{aligned}
$$

which are useful when the outermost relativistic image can be resolved from the rest. " $s$ " represents the angular separation between the first image and the limiting value of the images for $n \rightarrow \infty$. " $r$ " is the ratio between the flux of the first image and sum of the fluxes of the other images. Applying (85) and (87) one can show exact form of the equation (98) which without the negligible term $0<\zeta_{1} / D \ll 1$ becomes

$$
s \approx \theta_{\infty} e^{\left(c_{2}-2 \pi\right) / c_{1}}
$$

in which we defined

$$
\theta_{\infty}=\frac{u_{p s}}{\widetilde{d}_{O L}}
$$

and numerical value of $\widetilde{d}_{L O}$ is used here for Sgr A* observed galactic black hole as follows.

$$
\tilde{d}_{\mathrm{OL}}=\frac{d_{\mathrm{OL}}}{R_{S C H}}=\frac{2.47 \times 10^{17} \mathrm{~m}}{10^{10} \mathrm{~m}}=2.47 \times 10^{7} .
$$

One can infer that (99) can be rewritten as

$$
r=\frac{2 \mu_{1}}{\mu_{\text {tot }}^{\text {strong }}-2 \mu_{1}}
$$

which by inserting (94) and (95) reads

$$
r=\frac{e^{2 \pi / c_{1}}+e^{c_{2} / c_{1}}-e^{-2 \pi / c_{1}}+e^{c_{2} / c_{2}} e^{-4 \pi / c_{1}}}{1+e^{-2 \pi / c_{1}}-e^{c_{2} / c_{1}} e^{-4 \pi / c_{1}}}
$$

and its Taylor series expansion about $e^{-2 \pi / c_{1}} \rightarrow 0$ becomes

$$
r \approx e^{2 \pi / c_{1}}+e^{c_{2} / c_{1}}-1 .
$$

We plot diagrams of $c_{1,2}$ against $|q|$ in Figure 5 for $|q|<1$ and Figure 7 for $|q|>1$. They show increase of $c_{1,2}$ to large negative values in absence of NEM fields. While in presence of NEM fields $c_{1}$ takes on some positive values for $|q|<0.6$ and reaches to negative values for $0.6<|q|<1$. $c_{2}$ decreases by increasing $|q|<1$ in presence of NEM fields. For $|q|>1$ the diagrams of Figure 7 show increase of $c_{1}\left(c_{2}\right)$ to some positive (negative) values by decreasing $|q|>1$ in absence (presence) of NEM fields. $r(s)$ behaves as increasing (decreasing) function by raising $|q|>1$. In Figure 6 we see increase (decrease) for 



$$
\begin{gathered}
-\bar{\mu}_{\text {eff }}^{\text {weak }} \\
---\bar{\mu}^{\text {weak }}
\end{gathered}
$$$$
\begin{gathered}
\text { - } \\
-\bar{\mu}_{\mathrm{eff}}^{\text {weak }}
\end{gathered}
$$$$
y_{0}=10, \bar{\sigma}_{\text {eff }}^{\text {weak }}=-0.47, \bar{\sigma}^{\text {weak }}=+0.14
$$$$
y_{0}=10, \bar{\sigma}_{\text {eff }}^{\text {weak }}=-0.47, \bar{\sigma}^{\text {weak }}=+0.14, K=+1
$$
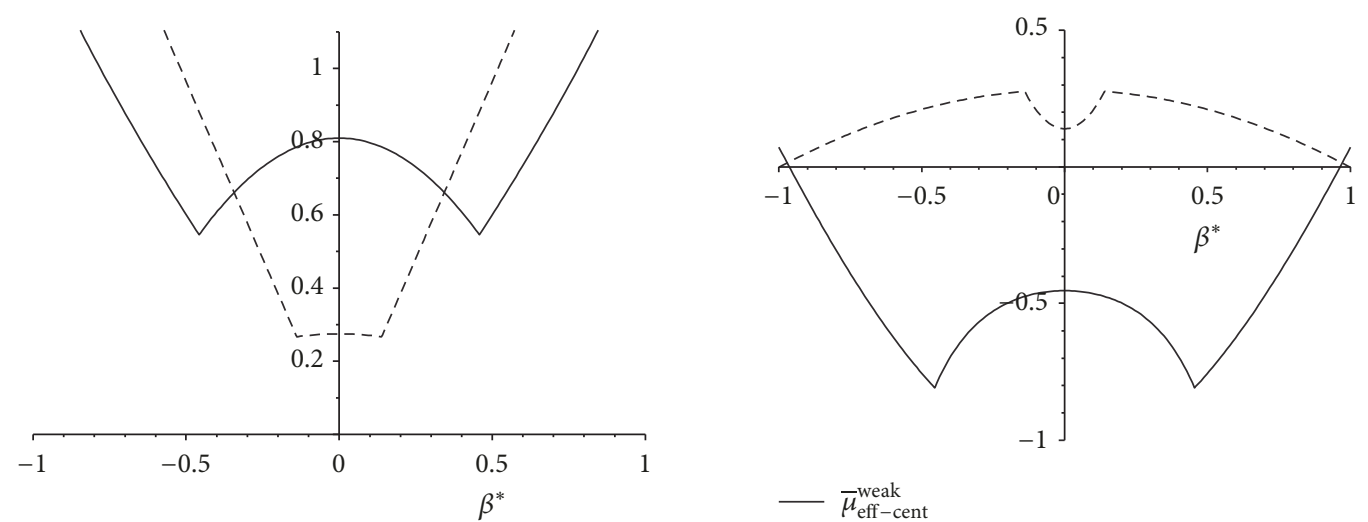

$$
\begin{aligned}
& ---\bar{\mu}_{\text {tot }}^{\text {weak }} \\
& -\bar{\mu}_{\text {tot-eff }}^{\text {weak }}
\end{aligned}
$$

$-\bar{\mu}_{\text {eff-cent }}^{\text {weak }}$

$$
---\bar{\mu}_{\text {cent }}^{\text {weak }}
$$

Figure 4: Diagrams of weak lensing image magnifications for $|q|<1$. 




In absence of nonlinear EM fields counterpart

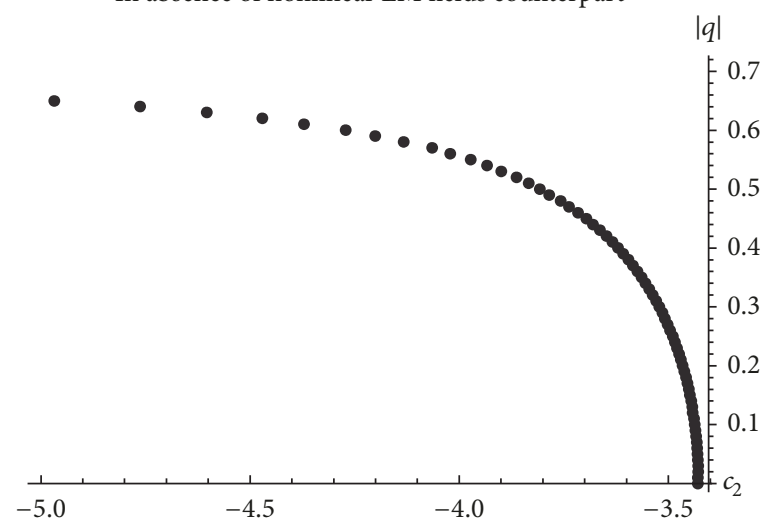

In presence of nonlinear EM fields counterpart



In presence of nonlinear EM fields counterpart

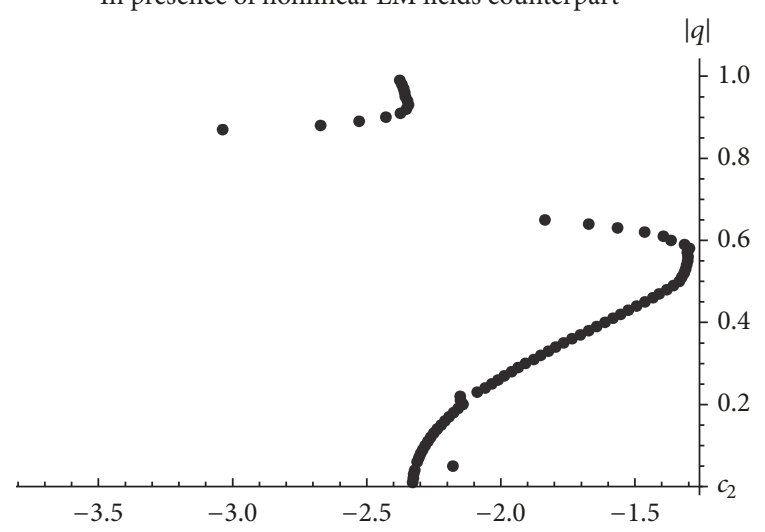

Figure 5: Diagrams for $c_{1}, c_{2}$ are plotted versus $|q|<1$.
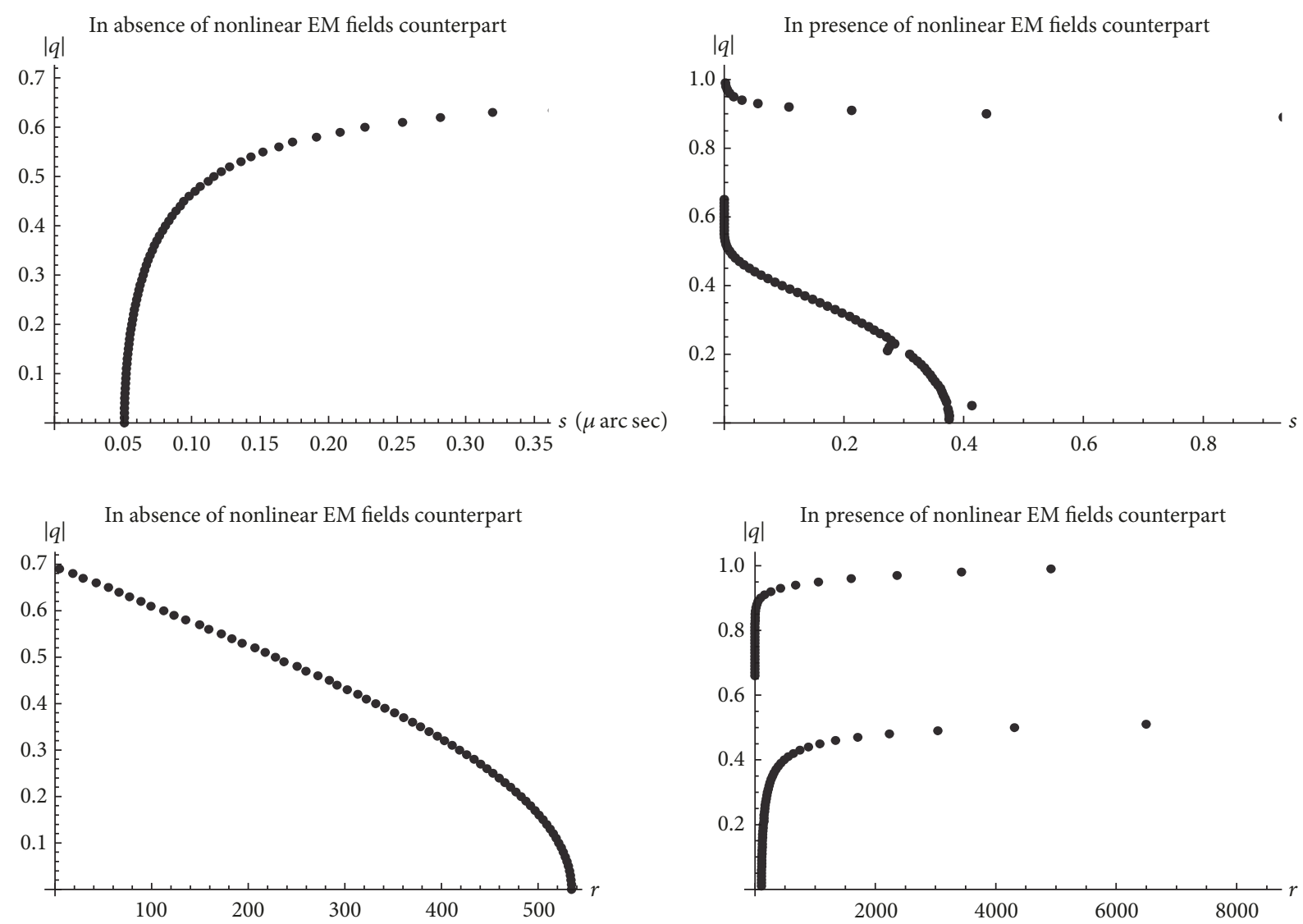

FIGURE 6: Diagrams for $s, r$ are plotted against $|q|<1$. 

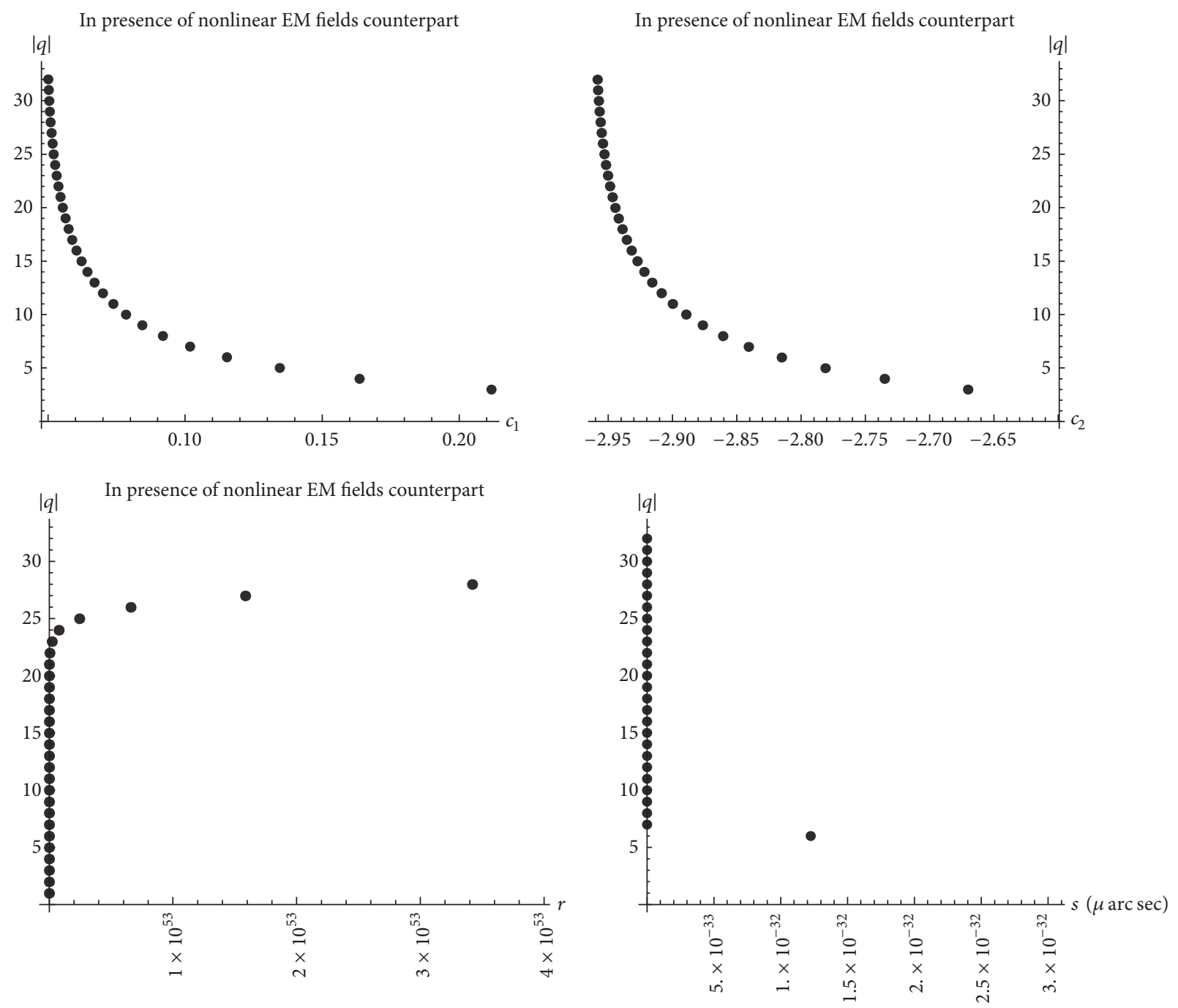

FIGURE 7: Diagrams for $c_{1}, c_{2}, s, r$ are plotted versus $|q|>1$.

$s$ by raising $|q|<1$ in absence (presence) of NEM fields. $r$ decreases (increase) by raising $|q|<1$ in absence (presence) of the NEM fields.

\section{Concluding Remark}

As a black hole solution of Born Infeld Einstein nonlinear Maxwell gravity we use ABG nonsingular charged black hole to study its gravitational lensing in WDL and SDL. We set our calculations to Sgr A* observed black hole date and study NEM field effects on the gravitational lensing. In short we obtained negligibility of NEM effects for large values of the black hole charge but not for its small values. Nonlinearity causes the photon sphere radius to be larger. Sign of deflection angle of bending light rays is changed just with NEM field effects with respect to state where the NEM field is absent. Einstein rings become larger and their magnifications become greater. Angular separation between the innermost and the outermost relativistic images decreases by increasing the charge parameter while their magnifications increase. As a subsequent work one can use strategy and results of this work to compare with results obtained by studying gravitational lensing of other nonsingular galactic black holes described in the introduction section and setting other observed black holes data.

\section{Conflicts of Interest}

The authors declare that there are no conflicts of interest regarding the publication of this paper.

\section{References}

[1] S. W. Hawking and G. F. R. Ellis, The Large Scale Structure of Space-Time, Cambridge University Press, London, UK, 1973.

[2] R. M. Wald, General Relativity, University of Chicago Press, 1984.

[3] R. M. Wald, "Gravitational collapse and cosmic censorship," in Black Holes, Gravitational Radiation and the Universe, vol. 100 of 
Fund. Theories Phys., pp. 69-85, Kluwer Academic Publishers, Dordrecht, The Netherlands, 1999.

[4] H. Ghaffarnejad, "Quantum field backreaction corrections and remnant stable evaporating Schwarzschild-de Sitter dynamical black hole," Physical Review D: Particles, Fields, Gravitation and Cosmology, vol. 75, no. 8, Article ID 084009, 2007.

[5] I. Dymnikova, "Spherically symmetric space-time with regular de Sitter center," International Journal of Modern Physics D, vol. 12, no. 6, pp. 1015-1034, 2003.

[6] A. A. Tseytlin, "On singularities of spherically symmetric backgrounds in string theory," Physics Letters B, vol. 363, no. 4, pp. 223-229, 1995.

[7] M. Cvetič, "Flat world of dilatonic domain walls," Physical Review Letters, vol. 71, no. 6, pp. 815-818, 1993.

[8] J. H. Horne and G. T. Horowitz, "Exact black string solutions in three dimensions," Nuclear Physics B, vol. 368, no. 2, pp. 444462, 1992.

[9] K. A. Bronnikov, H. Dehnen, and V. N. Melnikov, "Regular black holes and black universes," General Relativity and Gravitation, vol. 39, no. 7, pp. 973-987, 2007.

[10] S. Ansoldi, "Spherical black holes with regular center," in Proceedings of the BH2, Dynamics and Thermodynamics of Blackholes and Naked Singularities, Milano, Italy, May 2007, http://www.mate.polimi.it/bh2.

[11] J. Bardeen, Proceedings of GR5, Tbilisi, USSR, 1968.

[12] E. Ayón-Beato and A. Garcia, "The Bardeen model as a nonlinear magnetic monopole," Physics Letters B, vol. 493, no. 1-2, pp. 149-152, 2000.

[13] E. Ayon-Beato and A. Garcia, "Regular black hole in general relativity coupled to nonlinear electrodynamics," Physical Review Letters, vol. 80, p. 5056, 1998.

[14] E. Ayón-Beato and A. García, "New regular black hole solution from nonlinear electrodynamics," Physics Letters B, vol. 464, no. 1-2, pp. 25-29, 1999.

[15] E. Ayón-Beato and A. Garcia, "Non-singular charged black hole solution for non-linear source," General Relativity and Gravitation, vol. 31, no. 5, pp. 629-633, 1999.

[16] S. A. Hayward, "Formation and evaporation of nonsingular black holes," Physical Review Letters, vol. 96, Article ID 031103, 2006.

[17] J. C. S. Neves and A. Saa, "Regular rotating black holes and the weak energy condition," Physics Letters B, vol. 734, pp. 44-48, 2014.

[18] J. C. Neves, "Note on regular black holes in a brane world," Physical Review D: Particles, Fields, Gravitation and Cosmology, vol. 92, no. 8, 084015, 5 pages, 2015.

[19] M. Azreg-Aïnou, "Generating rotating regular black hole solutions without complexification," Physical Review D: Particles, Fields, Gravitation and Cosmology, vol. 90, no. 6, Article ID 064041, 2014.

[20] C. Bambi and L. Modesto, "Rotating regular black holes," Physics Letters B, vol. 721, no. 4-5, pp. 329-334, 2013.

[21] B. Toshmatov, B. Ahmedov, A. Abdujabbarov, and Z. Stuchlík, "Rotating regular black hole solution," Physical Review D: Particles, Fields, Gravitation and Cosmology, vol. 89, no. 10, Article ID 104017, 2014.

[22] S. G. Ghosh, "A nonsingular rotating black hole," The European Physical Journal C, vol. 75, no. 11, article no. 532, pp. 1-7, 2015.

[23] E. F. Eiroa and C. M. Sendra, "Regular phantom black hole gravitational lensing," Physical Review D: Particles, Fields, Gravitation and Cosmology, vol. 88, no. 10, Article ID 103007, 2013.
[24] L. Modesto and P. Nicolini, "Charged rotating noncommutative black holes," Physical Review D: Particles, Fields, Gravitation and Cosmology, vol. 82, no. 10, Article ID 104035, 2010.

[25] Z. Fan and X. Wang, "Construction of regular black holes in general relativity," Physical Review D: Particles, Fields, Gravitation and Cosmology, vol. 94, no. 12, 2016.

[26] B. Toshmatov, S. Zdenek, and A. Bobomurat, "Generic rotating regular black holes in general relativity coupled to nonlinear electrodynamics," Physical Review D, vol. 95, Article ID 084037, 2017.

[27] S. G. Ghosh, P. Sheoran, and M. Amir, "Rotating Ayón-BeatoGarcía black hole as a particle accelerator," Physical Review D: Particles, Fields, Gravitation and Cosmology, vol. 90, no. 10, Article ID 103006, 2014.

[28] Z. Stuchlik, "The motion of test particles in black-hole backgrounds with nonzero cosmological constant," Astronomical Institutes of Czechoslovakia. Bulletin, vol. 34, no. 3, pp. 129-149, 1983.

[29] Z. Stuchlik and M. Calvani, "Null geodesics in black hole metrics with nonzero cosmological constant," General Relativity and Gravitation, vol. 23, no. 5, pp. 507-519, 1991.

[30] Z. Stuchlík and S. Hledík, "Equatorial photon motion in the Kerr-Newman spacetimes with a non-zero cosmological constant," Classical and Quantum Gravity, vol. 17, no. 21, pp. 4541-4576, 2000.

[31] G. V. Kraniotis, "Gravitational lensing and frame dragging of light in the Kerr-Newman and the Kerr-Newman (anti) de Sitter black hole spacetimes," General Relativity and Gravitation, vol. 46, no. 11, 2014.

[32] M. Patil and P. S. Joshi, "Ultrahigh energy particle collisions in a regular spacetime without black holes or naked singularities," Physical Review D: Particles, Fields, Gravitation and Cosmology, vol. 86, no. 4, Article ID 044040, 2012.

[33] Z. Stuchlík, J. Schee, and A. Abdujabbarov, "Ultra-high-energy collisions of particles in the field of near-extreme KehagiasSfetsos naked singularities and their appearance to distant observers," Physical Review D: Particles, Fields, Gravitation and Cosmology, vol. 89, no. 10, Article ID 104048, 2014.

[34] A. García, E. Hackmann, J. Kunz, C. Lämmerzahl, and A. Macías, "Motion of test particles in a regular black hole spacetime," Journal of Mathematical Physics, vol. 56, no. 3, 032501, 20 pages, 2015.

[35] Z. Stuchlík and J. Schee, "Circular geodesic of bardeen and ayon-beato-garcia regular black-hole and no-horizon spacetimes," International Journal of Modern Physics D, vol. 24, no. 2, Article ID 1550020, 2015.

[36] Z. Stuchlík and J. Schee, "Optical effects related to Keplerian discs orbiting Kehagias-Sfetsos naked singularities," Classical and Quantum Gravity, vol. 31, no. 19, Article ID 195013, 2014.

[37] J. Schee and Z. Stuchlík, "Profiled spectral lines generated by Keplerian discs orbiting in the Bardeen and Ayòn-Beato-Garcìa spacetimes," Classical and Quantum Gravity, vol. 33, no. 8, Article ID 085004, 2016.

[38] A. Kehagias and K. Sfetsos, "The black hole and FRW geometries of non-relativistic gravity," Physics Letters B, vol. 678, no. 1, pp. 123-126, 2009.

[39] E. F. Eiroa, G. E. Romero, and D. F. Torres, "Reissner-Nordström black hole lensing," Physical Review D: Particles, Fields, Gravitation and Cosmology, vol. 66, no. 2, Article ID 024010, 2002.

[40] E. F. Eiroa and C. M. Sendra, "Gravitational lensing by a regular black hole," Classical and Quantum Gravity, vol. 28, no. 8, Article ID 085008, 2011. 
[41] S.-W. Wei, Y.-X. Liu, and C.-E. Fu, "Null geodesics and gravitational lensing in a nonsingular spacetime," Advances in High Energy Physics, vol. 2015, Article ID 454217, 2015.

[42] H. Ghaffarnejad and H. niad, "Weak gravitational lensing from regular Bardeen black holes," International Journal of Theoretical Physics, vol. 55, no. 3, pp. 1492-1505, 2016.

[43] V. Bozza, "Gravitational lensing in the strong field limit," Physical Review D: Particles, Fields, Gravitation and Cosmology, vol. 66, Article ID 103001, 2002.

[44] V. Bozza, "Gravitational lensing by black holes," General Relativity and Gravitation, vol. 42, no. 9, pp. 2269-2300, 2010.

[45] S. Sahu, K. Lochan, and D. Narasimha, "Gravitational lensing by self-dual black holes in loop quantum gravity," Physical Review D: Particles, Fields, Gravitation and Cosmology, vol. 91, no. 6, Article ID 063001, 2015.

[46] E. F. Eiroa and D. F. Torres, "Strong field limit analysis of gravitational retrolensing," Physical Review D: Particles, Fields, Gravitation and Cosmology, vol. 69, no. 6, 2004.

[47] E. F. Eiroa, "Braneworld black hole gravitational lens: strong field limit analysis," Physical Review D: Particles, Fields, Gravitation and Cosmology, vol. 71, no. 8, Article ID 083010, pp. 1-7, 2005.

[48] R. Genzel, F. Eisenhauer, and S. Gillessen, "The Galactic center massive black hole and nuclear star cluster," Reviews of Modern Physics, vol. 82, no. 4, 2010.

[49] S. Sahu, M. Patil, D. Narasimha, and P. S. Joshi, "Can strong gravitational lensing distinguish naked singularities from black holes?" Physical Review D: Particles, Fields, Gravitation and Cosmology, vol. 86, no. 6, Article ID 063010, 2012.

[50] R. R. Cuzinatto, C. A. M. de Melo, K. C. de Vasconcelos, L. G. Medeiros, and P. J. Pompeia, "Non-linear effects on radiation propagation around a charged compact object," Astrophysics and Space Science, vol. 359, no. 2, article no. 59, 2015.

[51] M. Novello, V. A. de Lorenci, J. M. Salim, and R. Klippert, "Geometrical aspects of light propagation in nonlinear electrodynamics," Physical Review D, vol. 61, Article ID 045001, 2000.

[52] N. Bretón, "Geodesic structure of the Born-Infeld black hole," Classical and Quantum Gravity, vol. 19, no. 4, pp. 601-612, 2002.

[53] H. Ghaffarnejad, "Classical and quantum Reissner-Nordström black hole thermodynamics and first order phase transition," Astrophysics and Space Science, vol. 361, no. 1, article no. 7, pp. 1-13, 2016.

[54] H. C. Ohanian, "The black hole as a gravitational "lens"," American Journal of Physics, vol. 55, no. 5, pp. 428-432, 1987.

[55] A. M. Ghez, S. Salim, N. N. Weinberg et al., "Measuring distance and properties of the milky way's central supermassive black hole with stellar orbits," The Astrophysical Journal, vol. 689, no. 2, pp. 1044-1062, 2008.

[56] F. Melia, The Black Hole at the center of Our Galaxy, Princeton University Press, Princeton, NJ, USA, 2003.

[57] F. Eisenhauer, R. Genzel, T. Alexander et al., "Sinfoni in the galactic center: young stars and infrared flares in the central light-month," The Astrophysical Journal, vol. 628, no. 1 I, pp. 246-259, 2005.

[58] J. Schee and Z. Stuchlík, "Gravitational lensing and ghost images in the regular Bardeen no-horizon spacetimes," Journal of Cosmology and Astroparticle Physics, vol. 2015, no. 6, article no. $048,2015$.

[59] E. F. Eiroa, "Gravitational lensing by Einstein-Born-Infeld black holes," Physical Review D: Particles, Fields, Gravitation and Cosmology, vol. 73, no. 4, Article ID 043002, 2006.
[60] C.-M. Claudel, K. S. Virbhadra, and G. F. Ellis, “The geometry of photon surfaces," Journal of Mathematical Physics, vol. 42, no. 2, pp. 818-838, 2001.

[61] S. Weinberg, Gravitation and Cosmology: Principle and Applications of the General Theory of Relativity, Wiley, New York, NY, USA, 1972.

[62] V. Bozza, "Comparison of approximate gravitational lens equations and a proposal for an improved new one," Physical Review D: Particles, Fields, Gravitation and Cosmology, vol. 78, no. 10, Article ID 103005, 2008.

[63] K. S. Virbhadra and G. F. R. Ellis, "Schwarzschild black hole lensing," Physical Review D: Particles, Fields, Gravitation and Cosmology, vol. 62, no. 8, Article ID 084003, 2000.

[64] B. S. Gaudi and A. O. Petters, "Gravitational microlensing near caustics. II. Cusps," The Astrophysical Journal, vol. 580, no. 1 I, pp. 468-489, 2002. 

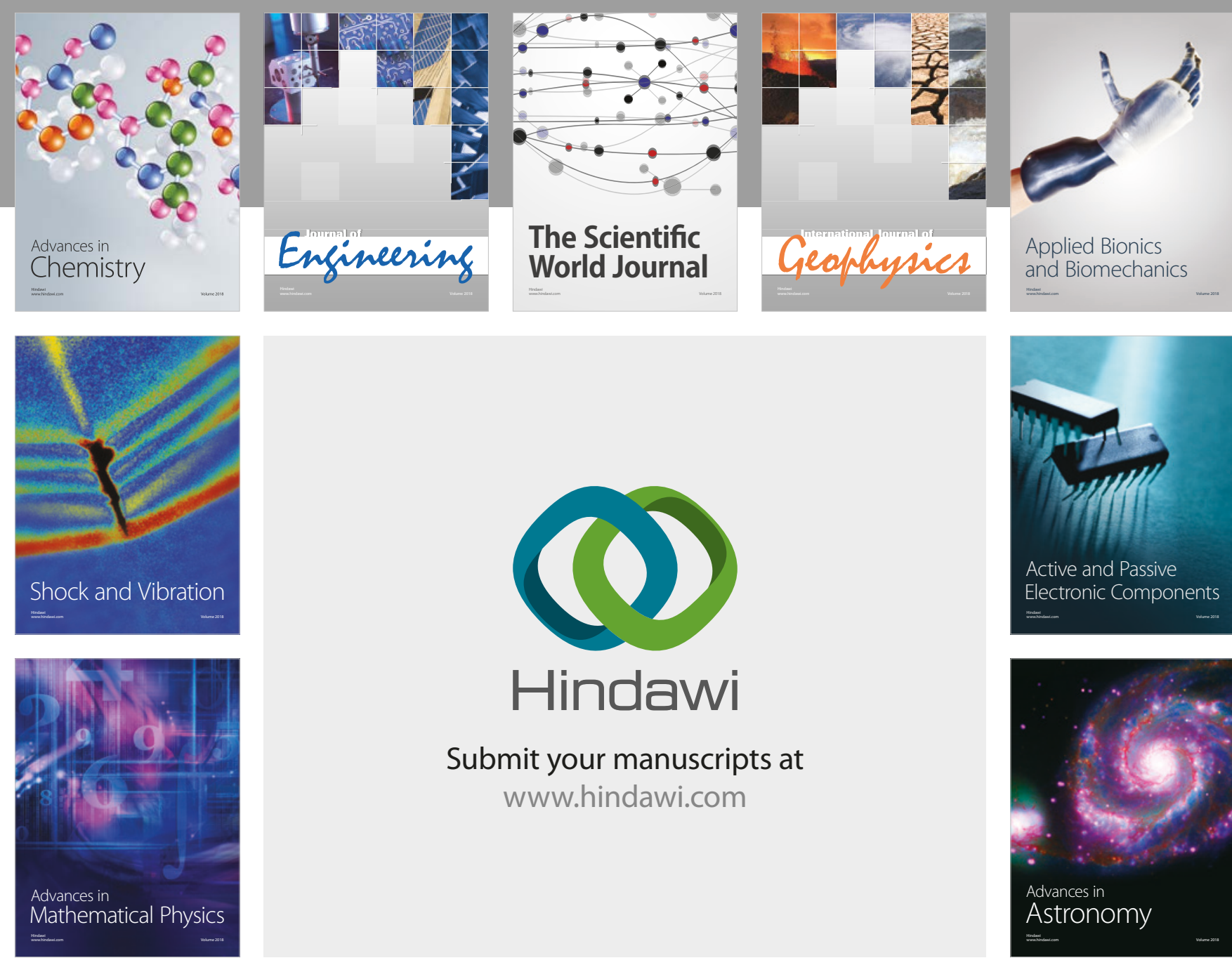

Submit your manuscripts at

www.hindawi.com

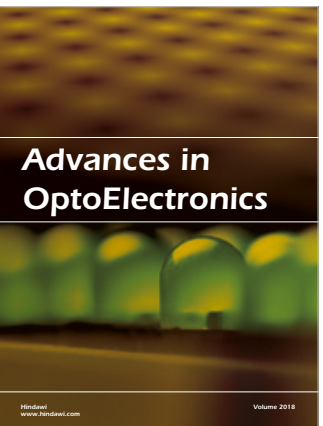

\section{Rotcting Machinery}
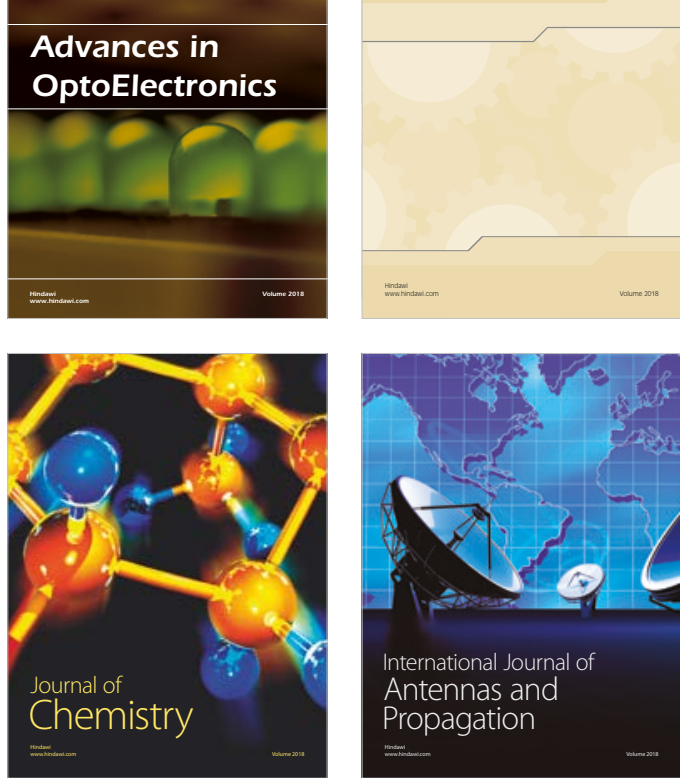



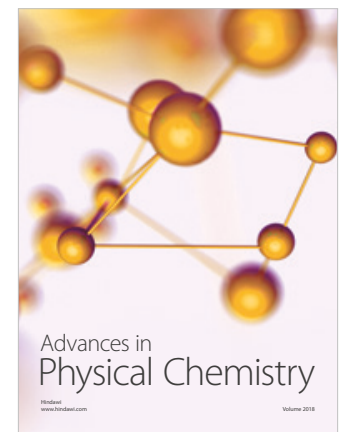

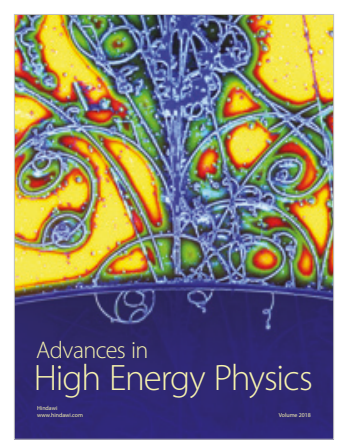

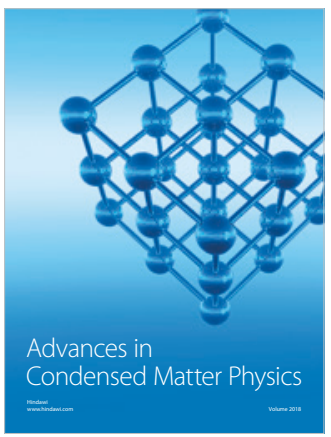

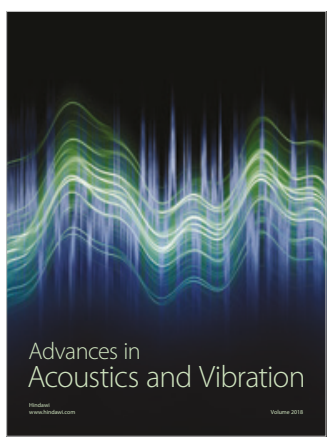


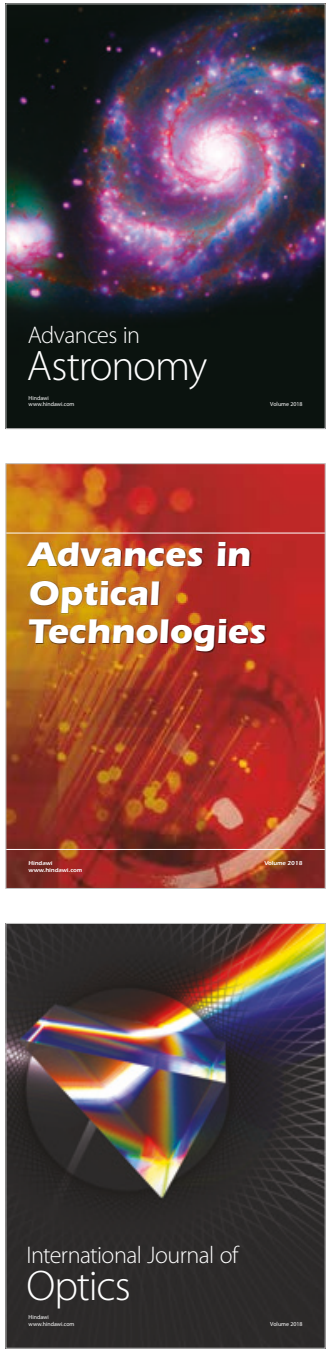